\title{
Activités
}

7-1 | avril 2010

Varia

\section{De la procédure in abstracto à la procédure « en acte »}

Le cas de la signification des actes authentiques par les clercs d'huissier de justice

From a procedure in abstracto to a procedure " in action ": the case of writs being served by bailiffs' clerks

\section{Damien Collard}

\section{OpenEdition}

Journals

Édition électronique

URL : http://journals.openedition.org/activites/2334

DOI : 10.4000/activites.2334

ISSN : $1765-2723$

Éditeur

ARPACT - Association Recherches et Pratiques sur les ACTivités

\section{Référence électronique}

Damien Collard, « De la procédure in abstracto à la procédure « en acte » », Activités [En ligne], 7-1 | avril 2010, mis en ligne le 15 avril 2010, consulté le 20 avril 2019. URL : http://journals.openedition.org/ activites/2334 ; DOI : 10.4000/activites.2334

\section{(c) (1) () $\Theta$}

Activités est mis à disposition selon les termes de la licence Creative Commons Attribution - Pas d'Utilisation Commerciale - Pas de Modification 4.0 International. 


\title{
De la procédure in abstracto à la procédure « en acte »: le cas de la signification des actes authentiques par les clercs d'huissier de justice
}

«Rien dans la règle ne prédétermine son application dans un cas particulier ( ). Ce qu'une règle implique est défini par des processus sociaux contingents »(Barnes, 1995).

\author{
Damien Collard \\ Université de Franche-Comté, 1 rue Claude Goudimel, 25030 Besançon cedex \\ damien.collard@univ-fcomte.fr
}

\begin{abstract}
From a procedure in abstracto to a procedure «in action»: the case of writs being served by bailiffs' clerks. This article shows that applying a procedure is not a mechanical (or simple) consequence of the procedure itself. It depends upon a series of practical tasks carried out in concrete situations. This idea is illustrated by the example of a legal procedure known as the serving of writs by bailiffs' clerks. The procedure has a general framework which must be respected in all circumstances. Nevertheless, observation shows that in practice, clerks are obliged to deal with and adapt to local circumstances and take advantage of opportunities which occur in their environment. Consequently, a distinction may be drawn between the procedure in abstracto - as a corpus of formal rules in a textbook and the procedure «in action» - as a series of practical tasks relying upon heterogeneous resources such as written rules, data provided by local contexts, and the clerks' experience and professional skills.
\end{abstract}

KEYWORDS

Serving writs, bailiffs' clerks, procedure, practice, skills, situation.

\section{Introduction}

En droit, la « signification » consiste en la remise physique d'un acte à son destinataire par un officier ministériel ou un agent spécialement habilité (Isnard, 2004). L'acte dont il est question ici est dit « authentique » car il émane d'un officier ministériel, c'est-à-dire d'un agent reconnu par l'Etat et qualifié pour produire de tels actes dans les formes attendues. Une fois l'acte produit, une copie va être remise à son destinataire. Là encore, c'est un officier ministériel, en l'occurrence un huissier de justice, qui opère. Celui-ci détient, en vertu de la loi, le monopole de la signification des actes authentiques. Mais dans la pratique c'est généralement un clerc d'huissier de justice qui mène à bien cette opération. Certains clercs (dits « clercs significateurs ») sont en effet spécialement chargés de « signifier» les actes et les décisions de justice, en les remettant en mains propres aux justiciables ou, à défaut, à un tiers.

Le métier de clerc d'huissier de justice n'a fait l'objet d'aucune étude. Dans le domaine juridique, les sociologues se sont avant tout intéressés à des professions plus «nobles »: les juges de la Cour de Cassation (Bancaud, 1989), les conseillers d'Etat (Latour, 2002), les avocats (Karpik, 1995 ; Milburn, 2002), les commissaires-priseurs (Quemin, 1997), les notaires (Suleiman, 1987; Thuderoz, 
1991), les huissiers de justice (Mathieu-Fritz, 2003, 2005, 2006 ; Thuderoz, 1991, op. cit.). Pourquoi ce désintérêt pour les clercs d'huissier de justice? Il tient avant tout à la nature des tâches qui leur sont confiées et au statut qu'ils occupent. Ce sont des exécutants, des "petites mains », à qui l'on confie des tâches peu valorisantes: tâches administratives réalisées au sein des études ou tâches liées à la signification des actes authentiques effectuées sur le terrain. Dans les deux cas, les clercs exécutent des procédures et effectuent un travail invisible et routinier qui repose sur le respect d'un certain nombre de règles et sur des «allants de soi $»^{1}$, un travail dont on ne voit bien souvent que le résultat (un courrier envoyé, un dossier complété, un acte signifié ). On est du côté de l'exécution du droit (de l'intendance) et non du côté de la conception du droit et des «théoriciens » ou «interprètes »du droit $^{2}$ (Bourdieu, 1986). De plus, les clercs n'existent que dans l'ombre des huissiers de justice. En effet, ils agissent pour le compte d'un huissier et sous sa responsabilité.

Mais que recouvre réellement le travail des clercs significateurs? Comment passe-t-on de la règle à la pratique? Que signifie suivre une procédure juridique et appliquer un certain nombre de règles de droit? Quel enseignement plus général peut-on tirer de ce cas particulier et que veut dire au juste « suivre une règle »? Dans le cadre de la procédure de signification des actes authentiques, des règles très précises doivent être respectées, sous peine de nullité de la procédure. La procédure de signification est donc un ensemble de règles qu'il faut appliquer strictement dans une situation déterminée. Mais comment cette procédure se déploie-t-elle dans des situations toujours particulières et contingentes? Car dire le droit ne suffit pas, encore faut-il qu'il soit suivi d'effets et que les informations parviennent aux justiciables (Deharo, 2005). Le droit doit en effet s'incarner dans des situations via des intermédiaires que constituent les huissiers de justice et les clercs. Cependant, comme nous le montrons à travers cet article, le suivi d'une procédure ne réside pas dans la procédure elle-même; c'est un accomplissement pratique qui prend appui sur des ressources hétérogènes: des règles, des données puisées dans le contexte local, une expérience et des compétences professionnelles. Toute une série d'opérations permettent à la procédure d'exister - non pas de manière abstraite (elle a une réalité objective dans la mesure où elle est constituée d'un ensemble de règles qui sont inscrites dans des textes) - mais en pratique, en référence à des situations particulières et dans des " cours d'action » (Theureau, \& Filippi, 1994). La procédure « en acte » pourra ainsi être distinguée de la procédure in abstracto.

Après avoir exposé le cadre théorique et méthodologique (l'enjeu et l'ancrage théorique de la recherche, le terrain et la méthodologie), l'activité des clercs d'huissier de justice sera décrite et analysée pour éclairer le passage de la procédure in abstracto à la procédure « en acte ».

\section{1.- Cadre théorique et méthodologie}

\section{1.- Enjeu et ancrage théorique de la recherche}

Cette recherche s'inscrit dans un ensemble de travaux qui portent sur les rapports entre les règles et la pratique. Pour la sociologie des professions et les sciences juridiques, le passage de la règle à la pratique constitue un épiphénomène, une question mineure et sans grand intérêt d'un point de vue théorique. A contrario, pour les sciences du travail et l'ethnométhodologie cette question est centrale, mais les approches diffèrent. Toute une veine de recherches menées dans le champ des sciences du travail met l'accent sur les tensions existantes entre les règles formelles et la pratique: le « travail réel » ne se réduirait jamais au « travail prescrit ». L'approche ethnométhodologique est quelque peu différente. Pour les ethnométhodologues, les règles sont intégrées à l'activité, n'existent qu'en situation, et les pratiques professionnelles sont envisagées comme des accomplissements pratiques.

1. Or, comme l'ont bien souligné les ethnométhodologues (Garfinkel, 1967a), « ce qui va de soi » ne se voit pas.

2. C'est-à-dire ceux qui participent à l'élaboration d'une théorie du droit (les universitaires) et à l'édification de la jurisprudence (les magistrats du siège et du parquet, les avocats...). 


\section{Le passage de la règle à la pratique : un épiphénomène}

Pour certains auteurs, la question de l'application des règles dans une situation particulière apparaît sur le mode de l'évidence et n'est pas pensée en tant que telle. Ces auteurs (sociologues ou juristes) ne s'intéressent pas aux pratiques situées des acteurs, ou de manière très marginale. C'est la position de la plupart des sociologues des professions qui ancrent leurs réflexions dans le fonctionnalisme (Merton, 1965; Parsons, 1968) ou dans le structuralisme génétique (Bourdieu, 1986, op. cit.). Ils rendent compte des comportements humains essentiellement à partir d'un ensemble de normes et de règles contraignantes et extérieures aux situations dans lesquelles les individus agissent. L'analyse du travail et le passage de la règle à la pratique constituent soit un angle mort, soit un épiphénomène. Dans le champ de la sociologie et de l'histoire des professions juridiques, on peut citer, par exemple, les travaux d'Halpérin sur les avocats et les notaires (Halpérin, 1996), de Quemin sur les commissaires-priseurs (Quemin, 1997, op. cit.), et dans une moindre mesure ceux de Mathieu-Fritz sur les huissiers de justice (Mathieu-Fritz, 2003, 2005, 2006, op. cit.). Quant aux juristes, ils ont défini très précisément ce en quoi consistaient la signification des actes authentiques et les règles à respecter en la matière (la procédure in abstracto). Les huissiers et les clercs doivent en effet suivre un canevas très précis et sont tenus de respecter les formalités en vigueur en matière de signification, sous peine de nullité de la procédure. Mais l'application des règles et des formalités liées à la signification des actes authentiques dans des situations concrètes n'est pas pensée en tant que telle. Les juristes ont également travaillé sur les missions, les prérogatives et les responsabilités de l'huissier de justice en tant qu'officier ministériel (Guinchard, \& Moussa, 2005 ; Guinot, 2004 ; Tilinski, 2002). Mais la pratique - c'est-à-dire ce que font réellement les huissiers (au sein de leur étude ou sur le terrain quand ils sont au contact des justiciables) et ce qu'ils font faire à d'autres (aux clercs) - ne relève pas du cadre d'analyse du juriste. Ce dernier concentre avant tout son attention et son regard sur la doctrine juridique et ses modalités d'élaboration (à travers notamment l'analyse de la jurisprudence), objet à ses yeux autrement plus noble et digne d'importance.

\section{Les tensions existantes entre les règles formelles et la pratique}

Toute une veine de recherches menées dans le champ des sciences du travail met en évidence les tensions existantes entre les règles formelles et la pratique. Ainsi, de nombreux ergonomes et sociologues du travail se sont intéressés aux pratiques professionnelles des travailleurs à partir des écarts irréductibles qui existent entre le «travail prescrit » et le «travail réel », entre la «tâche » et «l'activité ». Cette découverte scientifique majeure, qui est fondatrice de l'ergonomie de langue française (ou ergonomie de l'activité), a ouvert la voie à un débat fécond sur la part respective du « donné » et du « créé » dans le travail (Beguin, \& Clot, 2004) et à des travaux mettant à jour les compétences réelles des travailleurs (souvent masquées car invisibles et incorporées). Toute une littérature - qui va de l'ergonomie à la sociologie du travail en passant par la psychodynamique du travail (Dejours, 1995, 1998) - se réfère à la distinction entre le «travail prescrit » et le «travail réel ». Ces chercheurs décrivent et analysent, par le menu détail, l'activité des travailleurs, les écarts existants entre les règles officielles et la pratique, et reconstituent la raison d'être de ces écarts. Cantonnées à l'origine au monde de l'industrie dans un contexte de dénonciation des effets pervers engendrés par le taylorisme, ces recherches se sont ensuite étendues au monde des services via un ensemble de réflexions sur la relation de service dans les années quatre-vingt-dix et $2000^{3}$. Ce sont principalement les capacités d'adaptation des agents de base au contact du public (notamment leur capacité à ajuster la règle à la situation), leurs compétences, et les jeux individuels et collectifs qui se déploient autour des règles officielles qui sont mises en avant dans ces travaux de recherche ${ }^{4}$. Mais les tensions entre le

3. Goffman a été une source d'influence considérable pour les chercheurs s'intéressant à la relation de service (Gadrey, 1994 ; Joseph, 1995), dans la mesure où il a été le premier à avoir théorisé la relation de service (Goffman, 1968).

4. Voir par exemple, les travaux des ergonomes sur la relation de service, notamment ceux de: Caroly et Weill-Fassina (2007), Cerf et Falzon (2005), Djibo (2008), Falzon et Lapeyrière (1998), Leduc (2003), Valléry (2002, 2004), Valléry et Bonnefoy (1997). Voir également les travaux sociologiques de Bernard sur les caissières (Bernard, 2005), de Losego sur les agents administratifs (Losego, 2004), de Demazière et Mercier sur les facteurs (Demazière, \& Mercier, 
«prescrit » et le « réel » sont appréhendées différemment selon les auteurs. Pour certains, le « réel » s'oppose au « prescrit ». Ainsi, pour Dejours, le « réel est ce qui dans la tâche ne peut être obtenu par l'exécution rigoureuse du prescrit» (Dejours, 1995, op. cit., p. 45). Pour la psychodynamique du travail, travailler c'est nécessairement s'écarter des procédures et des règles officielles; le « réel » est donc subversif par rapport au «prescrit » et à l'organisation (officielle) du travail. D'autres chercheurs sont beaucoup plus nuancés. Le « travail réel » viendrait en quelque sorte compléter le « travail prescrit » (le « travail réel » est ici ce qui est réellement fait par le travailleur pour atteindre les objectifs fixés). Ainsi, Theureau, à travers son programme intitulé « cours d'action » (Theureau, 1992), braque le projecteur sur les capacités d'interprétation des agents: ces derniers interprètent constamment les situations dans lesquelles ils sont placés et réinterprètent la tâche à la lumière d'un certain nombre de contingences locales ${ }^{5}$. Clot et Faïta, quant à eux, mettent l'accent sur le travail d'organisation pris en charge par les collectifs de travail - le « genre du métier »-pour répondre aux prescriptions organisationnelles (Clot, \& Faïta, 2000). Selon eux, entre la tâche d'un côté et l'activité du sujet de l'autre, s'opère un travail de réorganisation/réinterprétation de la tâche par les collectifs de travail.

\section{Les pratiques professionnelles : des accomplissements pratiques}

Pour les ethnométhodologues, les règles sont constitutives de la pratique et n'existent qu'en situation. Il convient donc de prendre le respect des règles au sérieux en décrivant les opérations que ce respect engage. Dans cette perspective, les pratiques professionnelles sont appréhendées comme des accomplissements pratiques réalisés en situation (Garfinkel, 1967a, op. cit.) et les règles comme des ressources situées. Tout un pan des études ethnométhodologiques porte sur le travail et les pratiques professionnelles, en particulier les pratiques juridiques. Les premiers travaux de Garfinkel et de Sacks ont d'ailleurs été consacrés aux pratiques de différents acteurs du droit: les délibérations des jurés dans un tribunal (Garfinkel, 1948), les modes de raisonnement des enquêteurs d'un centre de prévention du suicide (Garfinkel, 1967b), le travail et les routines des juristes pour Sacks (Travers, \& Manzo, 1997). En outre, de nombreuses recherches de terrain anglo-saxonnes ont porté sur les pratiques en milieu judiciaire (dans les tribunaux ou dans d'autres institutions): sur les avocats de la défense (Sudnow, 1965), sur les policiers (Bittner, 1967), dans les négociations de plaidoiries (Maynard, 1984), au cours des audiences de conciliation (Dingwall, \& Greatbatch, 1994; Garcia, 1991), au sein des tribunaux pour mineurs (Cicourel, 1995), sur les compétences des avocats pénalistes (Travers, 1997), sur les pratiques pédagogiques des enseignants en droit (Burns, 1997), dans le cadre des procès de crimes violents (Komter, 1998) ${ }^{6}$. Par ailleurs, les travaux de Bruno Latour sur les pratiques professionnelles des conseillers d'Etat (Latour, 2002, op. cit.) peuvent être rapprochés de ces recherches ethnométhodologiques, même si celui-ci ne se réclame pas ouvertement de ce courant de pensée.

Dans la perspective ethnométhodologique, les règles de droit ne sont pas vues comme des abstractions formelles existant indépendamment de ce que les acteurs en font. Il s'agit de décrire le droit à travers les pratiques développées par les acteurs parties prenantes dans la production du droit. Il ne s'agit donc ni d'envisager les « défaillances » des pratiques au regard d'un modèle idéal (Dupret, 2001, op. cit.), ni de les opposer systématiquement aux règles officielles. Les règles de droit sont avant tout considérées comme partie intégrante d'une pratique professionnelle.

Dans la mesure où la finalité de notre recherche porte sur la procédure " en acte », les pratiques professionnelles des clercs significateurs seront envisagées comme des accomplissements pratiques. En complément, deux autres familles de pensée qui entretiennent des liens de parenté avec l'ethno-

2003), de Collard sur les médiateurs sous statut emploi-jeune de la SNCF (Collard, 2003), de Licoppe sur les agents commerciaux des centres d'appel (Licoppe, 2002).

5. Celui-ci va puiser une partie de son inspiration dans le courant de «l'action située » (Suchman, 1987).

6. En France, un certain nombre de ces travaux ont été diffusés il y a quelques années dans le cadre d'un numéro spécial de la revue Droit et Société consacré à l'ethnométhodologie et à l'analyse de conversation sous la direction de Baudouin Dupret (Dupret, 2001). 
méthodologie, et qui accordent une grande importance aux interactions sociales et aux situations dans lesquelles se trouvent placés les acteurs sociaux, seront mobilisées. Celle de l'action située tout d'abord, fondée sur les travaux de Lucy Suchman (Suchman, 1987, op. cit.), qui insiste notamment sur le cadre matériel dans lequel se déroule l'action. L'action, pour Suchman, est construite en situation et ne se réduit pas à une simple exécution de règles, dans la mesure où elle dépend étroitement des circonstances matérielles et sociales. Agir, pour Suchman, c'est s'adapter au contexte local, aux circonstances, gérer les contingences, et saisir les opportunités présentes dans l'environnement. Celle de l'analyse dramaturgique ensuite, fondée sur les travaux d'Erving Goffman (Goffman, 1973, 1974), pour qui le monde social est un théâtre. Ce dernier s'est notamment intéressé au rôle régulateur joué par la civilité dans les interactions sociales. Le paradigme dans lequel notre recherche s'inscrit peut être qualifié de « situationniste », en référence au « situationnisme méthodologique » mis en avant par Isaac Joseph (Joseph, 1998).

\section{2.- Présentation du terrain : un bureau de signification d'une grande ville française}

L'étude réalisée sur la mise en œuvre de la signification des actes authentiques et les pratiques professionnelles des clercs significateurs s'inscrit dans une recherche plus large menée sous la direction de Béatrice Fraenkel (intitulée «Pratiques juridiques et écrits électroniques: la signature dans la société de l'information ») dans le cadre du programme «Société de l'information» du CNRS (2002-2004) et soutenue par le Groupement d'Intérêt Public (Mission de recherche « Droit et Justice ») du Ministère de la Justice ${ }^{7}$.

\section{Présentation de la structure: le «Bureau»}

Cette étude a été menée au sein d'un bureau de signification implanté dans une grande ville française (nommé le «Bureau » dans la suite de l'article). On trouve des bureaux de signification à Paris, en banlieue parisienne (Val-de-Marne, Hauts-de-Seine, Seine Saint-Denis) et dans la plupart des grandes villes de province (Marseille, Bordeaux, Nice ). Ces bureaux sont des structures anciennes (certains ont plus d'un siècle).

L'objectif du fondateur du Bureau était de « simplifier le travail par la concentration des efforts de tous sur un point commun: l'exactitude absolue dans la remise des copies aux intéressés ». La raison d'être du Bureau, précisée dans ses statuts, est de « réduire au bénéfice des huissiers de justice et par l'effort commun de ceux-ci, le prix de revient de certaines prestations relatives à l'exercice de leur profession, notamment la signification des actes d'huissiers de justice ». Ainsi, les huissiers de justice ont la possibilité, moyennant paiement, de déléguer au Bureau la signification de leurs actes authentiques. Ce sont les clercs significateurs du Bureau qui doivent alors signifier ces actes auprès des justiciables. Mais ils agissent pour le compte d'un huissier de justice et sous sa responsabilité.

Le statut du clerc significateur assermenté a été défini par la loi du 27 décembre 1923. Avant cette loi, les huissiers de justice devaient théoriquement signifier eux-mêmes tous leurs actes. Dans la pratique, ils sous-traitaient déjà cette tâche.

Outre la signification des actes authentiques, le Bureau assure aussi gratuitement un certain nombre de services pour le compte des huissiers, comme le courrier par exemple (entre les études, avec les avocats, avec les tribunaux).

Le Bureau est géré au quotidien par un gérant (qui a le statut de salarié), un ancien huissier de justice, qui a notamment pour mission d'organiser et de contrôler l'activité des clercs significateurs, et qui est responsable de la gestion du personnel.

7. Cette recherche a notamment donné lieu à la rédaction de deux rapports: l'un à destination du CNRS (Fraenkel, Pontille, Collard, Deharo, \& Blanchette, 2004) et l'autre à destination du GIP du Ministère de la Justice (Fraenkel, Pontille, Collard, \& Deharo, 2005). 


\section{Le travail de signification des actes authentiques et les différentes catégories de clercs}

Le travail de signification des actes authentiques ne se réduit pas aux tournées effectuées par les clercs et aux rencontres avec les justiciables. Il commence en amont, lors de la préparation de la tournée, dans les locaux du Bureau. En effet, tous les matins, un membre de l'encadrement du Bureau distribue aux différents clercs les plis à signifier. Ces derniers doivent alors organiser leur tournée pour la journée et planifier leur itinéraire. En outre, après leur tournée, dans les locaux du Bureau ou une fois chez eux, les clercs doivent rédiger un compte rendu pour chacun des actes signifiés ${ }^{8}$. Une journée type de travail se décompose donc en trois temps: (1) la préparation de la tournée; (2) la tournée; (3) la rédaction des compte-rendus d'activité.

Il existe plusieurs types de clercs significateurs au sein du Bureau. Tandis que les « doubleurs » (les

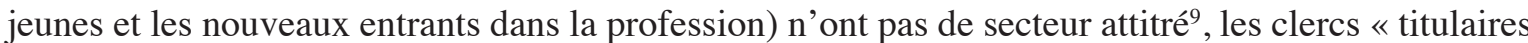
de tournée » (les plus expérimentés, ceux qui bénéficient d'une certaine ancienneté dans la structure) prennent en charge un secteur géographique donné. Par ailleurs, on peut opérer une distinction plus fine parmi les clercs «titulaires de tournée » entre ceux dont la tâche principale est de signifier les actes authentiques au domicile des justiciables et les « chefs de secteur ». Outre la signification des actes authentiques, ces derniers doivent effectuer certaines tâches administratives (organiser les plannings, établir la fiche journalière de secteur ) et ont des responsabilités organisationnelles (coordonner le travail de plusieurs « doubleurs », contrôler les tournées et les équilibrer ) et managériales (faciliter la socialisation des nouveaux venus dans la profession et leur inculquer le respect des règles). La rémunération des clercs significateurs est constituée d'une partie fixe (qui est fonction de l'ancienneté et du niveau de responsabilité) et d'une partie variable (qui dépend du nombre d'actes signifiés $)^{10}$.

\section{3.- Méthodologie : collecte et analyse des données}

\section{L'accès au terrain}

Dans la mesure où l'objectif de cette recherche était d'étudier l'activité de signification des actes authentiques (la procédure « en acte »), une observation fine du travail des clercs significateurs a été réalisée. Les clercs ont été observés pendant leurs tournées, mais aussi au moment de la préparation de celles-ci ${ }^{11}$.

Les données recueillies doivent être resituées dans leur contexte, dans la mesure où la présence d'un chercheur sur le terrain n'est pas neutre. Les acteurs attribuent en effet une place au chercheur et ajustent leur comportement en conséquence (Favret-Saada, 1978). Les clercs significateurs suivis sur le terrain avaient été sélectionnés au préalable par le gérant du Bureau selon deux critères: (1) le professionnalisme (les clercs les plus « professionnels » étaient pour le gérant ceux qui respectaient rigoureusement les règles de signification) et (2) le volontariat (ils devaient en effet accepter la présence d'un chercheur à leur côté). Seule l'activité des clercs expérimentés «titulaires de tournée »a donc fait l'objet d'observations. Parmi eux, un clerc était « chef de secteur » et jouait un rôle clé dans la socialisation et la formation des nouveaux entrants.

L'objet de l'enquête et la démarche de recherche ont été présentés à chacun des clercs volontaires.

8. La plupart des clercs préfèrent effectuer ce travail chez eux.

9. Ces derniers sont amenés à « tourner » sur les différents quartiers; tout dépend de la charge de travail du Bureau, des absences, et des périodes de l'année. Ce n'est généralement qu'au bout de plusieurs années qu'un « doubleur » peut espérer devenir titulaire d'une tournée (cela est notamment conditionné par les départs en retraite des plus âgés). Il y a ici un parallèle à faire avec les facteurs débutants de la Poste, ceux que l'on nomme les « rouleurs » et qui n'ont pas de territoire bien défini (Demazière, \& Mercier, 2003, op. cit.).

10. Au moment de l'enquête, les clercs significateurs du Bureau devaient signifier au moins 897 actes par mois (pour justifier le versement de leur salaire de base), soit 41 actes par jour travaillé. La moyenne s'établissait à 57 actes par jour, mais les clercs les plus « performants » signifiaient en moyenne une centaine d'actes par jour.

11. Les observations se sont étalées sur quinze jours. Par contre, l'activité «post tournée » n'a pas pu être observée. 
Il leur a également été précisé que l'objectif de cette enquête était de mieux connaître la « réalité du terrain » pour comprendre « de l'intérieur » leur métier. En outre, les retombées éventuelles de cette enquête ont été mises en avant pour légitimer la démarche de recherche: faire remonter à la hiérarchie les difficultés rencontrées sur le terrain, améliorer les formations existantes, faire reconnaître l'utilité du métier. Enfin, il a été précisé à chacun des clercs que cette recherche était commanditée par le CNRS et le Ministère de la Justice et qu'un rapport d'étude devait être remis au dirigeant du Bureau à la fin de l'enquête.

La présence d'un chercheur sur le terrain incitait donc les clercs à mettre en avant leurs «bonnes pratiques », celles qui sont congruentes avec les règles formelles en matière de signification des actes authentiques. Il y a là un effet induit par le dispositif de recherche. Cependant, c'est un effet induit « heureux », concordant avec l'objet de la recherche, puisqu'il s'agissait d'étudier l'activité sousjacente au déploiement d'une procédure juridique ${ }^{12}$.

\section{Dispositif méthodologique}

Plusieurs clercs ont été accompagnés, et ce dans différents quartiers, de manière à multiplier les points de vue et répertorier une grande variété de situations de travail. La sociologie du quartier et la configuration des lieux déterminent pour partie les types de situations rencontrées par les clercs. Les échanges verbaux entre les clercs et les personnes avec qui ils sont en contact (justiciables, gardiens...) ont été enregistrés via un dictaphone, retranscrits (dans un journal de terrain) et analysés, de même que les commentaires faits par les clercs a posteriori. Ils ont en effet apporté des éclairages très utiles sur les situations rencontrées (sur les cas qu'ils traitaient, sur les difficultés issues de la pratique, sur le contexte ) et se sont efforcés d'expliciter leur activité (en justifiant leurs actions et en les replaçant dans des chaînes d'actions plus larges dans lesquelles de multiples acteurs interviennent...). Ces commentaires faits a posteriori permettent de décrypter les stratégies qu'ils déploient en situation «à toutes fins pratiques » (Garfinkel, 1967a, op. cit.). Cette manière de procéder, qui est familière à l'ergonomie de l'activité, est étrangère à la démarche ethnométhodologique, puisque pour les ethnométhodologues les discours faits a posteriori par les membres d'un groupe sur leur activité (nécessairement située) ne permettent pas de rendre compte de cette activité (Relieu, Salembier, \& Theureau, 2004). Il s'agit pourtant d'un matériau langagier dont il serait dommage de se priver ${ }^{13}$.

Différents niveaux de discours ont donc été enregistrés et analysés. Le dictaphone utilisé pour les besoins de l'enquête était dissimulé dans l'une de nos poches et ne pouvait pas être perçu par les justiciables ${ }^{14}$. Les situations et les discours analysés peuvent donc être qualifiés de « naturels », exceptés les commentaires faits par les clercs après-coup.

En complément, des entretiens semi-directifs auprès du gérant et de l'encadrement de proximité ont été menés. L'objectif était de recueillir des informations sur le travail prescrit, l'organisation du travail, les démarches entreprises par l'encadrement de proximité pour contrôler et surveiller l'activité des clercs significateurs, et les modalités de gestion du personnel.

Enfin, certains documents ont été analysés (fiches de poste, notes de service, manuel du clerc significateur, rapports d'expertise établis par des consultants ). La finalité de cette étude documentaire était de mieux appréhender à la fois le contexte organisationnel et l'ensemble des règles qui régissent le travail des clercs significateurs (la procédure in abstracto).

Les analyses qui vont suivre reposent donc sur des données primaires, à savoir des observations de terrain et des entretiens, mais aussi sur des sources secondaires, constituées de données internes

12. Il existe néanmoins des pratiques de travail transgressives. Ainsi, selon un cadre du Bureau, sur les soixante-dix clercs du Bureau une dizaine étaient surveillés de près car ils ne respectaient pas scrupuleusement les règles et formalités qui régissent la procédure de signification des actes authentiques.

13. D'un point de vue méthodologique, Theureau propose ainsi de coupler méthodes d'observation et méthodes de verbalisation (Theureau, 1992, op. cit.).

14. Le gérant du Bureau avait au préalable donné son accord. Mais aucune affaire de justice ne devait être commentée et l'anonymat des personnes devait être respecté à tous les stades de la recherche. D'autre part, les clercs ont donné leur accord avant tout enregistrement. 
émanant de l'organisation étudiée.

\section{2.- De la procédure in abstracto à la procédure « en acte »: comment passe-t-on de la règle formelle à la pratique?}

Le passage de la procédure in abstracto à la procédure « en acte » ne va pas de soi. Il repose sur une série d'accomplissements pratiques réalisés en situation et sur le déploiement de certaines compétences professionnelles. Les principales règles et formalités à respecter en matière de signification des actes authentiques (la procédure in abstracto) seront dans un premier temps explicitées. Celles-ci orientent fortement les pratiques des clercs significateurs. Cependant, le respect apparent des règles n'épuise pas l'activité des agents (Salembier, \& Zouinar, 2004; Theureau, Filippi, Saliou, \& Vermersch, 2002). L'activité de signification des actes authentiques (la procédure "en acte ») et les compétences mobilisées par les clercs seront donc analysées dans un second temps.

\section{1.- La procédure in abstracto : les règles et formalités à respecter en matière de signification des actes authentiques}

Ces règles et formalités sont définies de manière très précise par les textes de loi : par le Nouveau Code de Procédure Civile (NCPC) pour la signification en matière civile (article 653 et suivants du NCPC), par le Code de Procédure Pénale (CPP) pour la signification en matière pénale (article 555 et suivants du $\mathrm{CPP}$ ). Ces textes définissent les règles qui régissent la signification des actes authentiques et contiennent des avis rendus par la jurisprudence (précisant les conditions et les principes d'application de ces règles). C'est à la lumière de ces règles et de la jurisprudence que les juges apprécient les pratiques professionnelles des clercs significateurs (notamment en cas de faute: triche, malveillance, manquement, négligence).

\section{Le manuel du clerc significateur}

Pour former et professionnaliser les clercs significateurs, la Chambre Nationale des Huissiers de Justice a édicté le manuel du clerc significateur, support destiné à l'ensemble des clercs significateurs qui exercent au sein d'une étude d'huissier ou d'un bureau de signification. Ce manuel est régulièrement révisé et actualisé en fonction des évolutions législatives et jurisprudentielles (au moment de l'enquête la version utilisée au sein du Bureau était la cinquième édition, celle de 1991). Ce manuel a une visée pédagogique: il est utilisé par les instituts de formation (comme l'Ecole Nationale de Procédure) et par les bureaux de signification en vue de former les nouveaux entrants dans la profession. Il expose les fondements du droit français (l'organisation judiciaire, la classification des juridictions, le rôle des magistrats, de l'huissier de justice), présente la place et le rôle du clerc significateur (attributions, responsabilités), définit l'acte de l'huissier de justice (sa portée, ses caractéristiques, sa forme), et décrit de manière précise les règles et les formalités à respecter en matière de signification des actes authentiques (en reprenant et en explicitant les dispositions contenues dans le NCPC et le $\mathrm{CPP}$ ). Il a donc également une visée prescriptive et constitue un « document prescripteur » (Leplat, 2004). L'encadrement et les formateurs, qui utilisent ce manuel à des fins pédagogiques (formateurs) ou de contrôle (encadrement), constituent, quant à eux, des « relais prescriptifs » essentiels (Denis, 2007). Enfin, dans la mesure où il définit un ensemble de « bonnes pratiques », il fait office de guide méthodologique et déontologique. Il vise en effet à orienter les comportements et les pratiques professionnelles en dispensant aux clercs des conseils pratiques et en valorisant certaines attitudes (par exemple, sont mis en avant l'honnêteté, la discrétion, la courtoisie, ou encore le tact). Au sein du Bureau, ce manuel est utilisé par l'encadrement pour rappeler les clercs à leurs devoirs, aussi bien sur le plan légal - ils doivent impérativement respecter les règles de signification - que sur le plan comportemental.

Dans la préface du manuel du clerc significateur (cinquième édition), Marcel Dymant, ancien Prési- 
dent de la Chambre Nationale des Huissiers de Justice, précise que le terme de «signification » recouvre deux idées: (1) celle d'informer et (2) celle de révéler le caractère expressif d'un fait ou d'un événement. La mission du clerc significateur consiste donc non seulement à transmettre un acte à un justiciable (si possible en mains propres) mais aussi à souligner la portée de celui-ci et son caractère solennel. Ainsi, même s'il est d'usage de dire que le clerc n'a pas à «commenter » les actes qu'il remet, il est en revanche tenu d'informer le destinataire d'un minimum d'éléments, les justiciables étant en effet des profanes. Par exemple, selon le manuel du clerc significateur, lorsqu'une personne reçoit une assignation devant le tribunal de grande instance et qu'elle est perplexe, rien n'empêche le clerc de lui indiquer que de toute urgence il serait bon qu'elle consulte un avocat.

\section{Les points de passage obligés en matière de signification des actes authentiques}

Selon les règles en vigueur ${ }^{15}$, le clerc doit tout entreprendre pour remettre la copie de l'acte à la personne même de l'intéressé et doit rendre compte de toutes les démarches qu'il a entreprises auprès de l'huissier de justice concerné. C'est le principe de la «signification à personne »; celui-ci est impératif. Lors de son assermentation, le clerc s'engage solennellement à respecter la procédure de signification des actes authentiques. S'il s'avérait que le clerc n'a pas respecté le principe de la « signification à personne »-s'il s'est montré laxiste, négligeant, s'il a manqué de persévérance - il peut être sanctionné lourdement par sa hiérarchie. Il existe ainsi, au sein du Bureau, toute une palette de sanctions, qui peuvent aller du simple avertissement (en cas de négligence ou de faute légère) au licenciement (en cas de faute grave, de triche avérée ). Dans le cas où la « signification à personne » est possible, il doit noter dans le « parlant à » (fiche annexée à l'acte sur laquelle il rédige son compte rendu) le lieu où la personne a été « touchée » (domicile, lieu de travail $)^{16}$. Ce n'est qu'à défaut de domicile connu que la signification peut être faite au lieu de la résidence du destinataire (son lieu de travail par exemple). Au domicile ou à la résidence, la copie peut être remise : à l'intéressé lui-même (s'il est présent dans les lieux); sinon à toute personne présente à la double condition qu'elle accepte de recevoir la copie, et qu'elle décline ses nom, prénom et qualité; à défaut, au gardien; en dernier lieu, à tout voisin. Le « parlant à » doit, quant à lui, préciser les nom, prénom et qualité déclarés et les démarches effectuées par le clerc. Dans le cas d'absence momentanée, ou d'impossibilité de remise, ou de refus, le clerc est tenu de remettre la copie de l'acte à la mairie le jour même, ou au plus tard le premier jour où les services de la mairie sont ouverts au public. Ces règles doivent être impérativement respectées. Enfin, dans tous les cas où la signification à la personne même de la partie intéressée s'est avérée impossible, certaines formalités annexes à la signification proprement dite doivent être effectuées. Elles consistent en un avis de passage (déposé par le clerc dans la boîte aux lettres du destinataire) et l'envoi d'une lettre simple contenant une copie de l'acte (formalité exécutée par l'huissier de justice ou par un de ses collaborateurs à l'étude). Ces règles structurent l'activité des clercs et imposent le respect d'un certain nombre d'étapes. Les clercs sont ainsi tenus de suivre un canevas très précis.

\section{2.- La procédure « en acte »: une approche par les pratiques et les compétences professionnelles des clercs significateurs}

Les règles et les formalités décrites ci-dessus sont généralement scrupuleusement respectées par les clercs. Mais le respect de celles-ci suppose un travail et n'est possible que parce que les clercs s'appuient sur un certain nombre de ressources (puisées dans leur environnement) et qu'ils mobilisent leurs compétences (qui dépassent très largement le fait d'avoir intériorisé les règles de signification $^{17}$ ). Il faut donc inclure dans l'analyse des éléments hétérogènes qui ne sont pas issus des règles formelles, mais qui s'enracinent dans une pratique professionnelle et qui constituent autant de res-

15. Il s'agit là des règles principales (les règles générales), celles qui encadrent la procédure de signification en matière civile; elles doivent être impérativement respectées, sous peine de nullité de la procédure.

16. Le manuel du clerc significateur donne quelques exemples: «Parlant à sa personne ainsi déclarée, trouvée au restaurant Dupont, $\mathrm{n}^{\circ}$ 6, rue Saint Jacques à Paris »; « Parlant à sa personne ainsi déclarée, rencontrée à son domicile »...

17. Les règles formelles ne sont en fait qu'une ressource parmi d'autres. 
sources qui sont au fondement de l'activité des clercs significateurs.

Dans la pratique, les clercs doivent planifier leur travail et organiser leurs tournées, interagir avec un certain nombre de personnes (justiciables, voisins, gardiens ), interpréter une situation sur la base des informations et des instructions qu'ils ont reçues des études d'huissier et des éléments qu'ils peuvent recueillir in situ. Pour ce faire, ils mobilisent leurs compétences professionnelles. Outre leurs connaissances et compétences juridiques, ils mobilisent des compétences spatiales, cognitives, et relationnelles. En situation, ces compétences sont étroitement enchevêtrées. Elles seront étudiées au regard des deux caractéristiques principales de l'activité des clercs significateurs. Premièrement, les clercs doivent « énacter » leur environnement (Weick, 1977, 1988) et «l'asservir» (Kirsh, 1995) pour pouvoir agir efficacement. En effet, l'action des clercs consiste en partie à créer un environnement propice au déploiement de la procédure de signification des actes authentiques. Deuxièmement, la civilité joue un rôle clé dans leur travail, dans la mesure où elle est au cœur de leur activité et au fondement de leur professionnalité.

\section{« Enacter » et « asservir » son environnement}

Karl Weick s'est inspiré des travaux menés en sciences cognitives par Francisco Varela sur l' «énaction » (Varela, 1989). Dans la perspective dessinée par Varela, « le concept d'enaction résume l'idée qu'à chaque instant, l'acteur « fait émerger » le monde de son action en relation avec son engagement dans l'environnement et que l'action et la cognition sont inséparables » (Leblanc, Ria, Dieumegard, Serres, \& Durand 2008, p. 62). Pour Weick (1988), l'« enactment perspective » renvoie simultanément à un processus ( « enactment») et à un résultat (« enacted environment»). Les individus jouent ainsi un rôle actif dans la «construction » de leur environnement. Ce faisant, ils construisent un environnement « énacté » qui va guider et faciliter leurs actions. Cette perspective peut être rapprochée de l'idée d' " asservissement» de l'environnement mise en évidence par Kirsh pour qualifier l'activité des experts (Kirsh, 1995, op. cit.). Les experts « asservissent » leur environnement dans la mesure où ils le structurent et l'aménagent pour pouvoir agir efficacement ${ }^{18}$. Les clercs significateurs sont amenés, eux aussi, à organiser et à structurer leur environnement, à le modeler pour l'adapter aux finalités poursuivies.

Le processus d'enactment est particulièrement visible au moment de la préparation de la tournée, tâche effectuée le matin dans les locaux du Bureau. Ce moment est très important car il conditionne le trajet qui sera effectué durant la journée, donc la charge de travail. Une tournée bien préparée en amont facilite en aval le travail de signification proprement dit. Préparer sa tournée consiste essentiellement à trier et à classer les plis qui contiennent les actes authentiques. Les clercs doivent anticiper et optimiser le trajet qu'ils feront dans la journée en tenant compte du nombre d'actes à signifier, des lieux où ils doivent se rendre, des caractéristiques spatiales du quartier correspondant à leur tournée, des contraintes émanant des études d'huissier (par exemple certains actes authentiques, qualifiés de « derniers jours », doivent impérativement être signifiés dans la journée), et des contingences locales... Pour ce faire, ils s'appuient sur un ensemble de ressources qui sont, selon les cas, personnelles ou partagées. Par exemple, certains clercs organisent leur tournée « en arc de cercle». Cette technique (qui n'est cependant pas utilisée par tous les clercs) est connue des plus anciens; elle s'est transmise au sein du groupe de pairs. Ceux qui l'utilisent disent ainsi limiter leurs déplacements au strict minimum. Ils peuvent, si besoin est, revenir rapidement sur un lieu où ils sont déjà passés (s'ils ont oublié de signifier un acte authentique par exemple). Structurer son environnement spatial en effectuant une tournée « en arc de cercle » est ainsi un gage de performance. Évidemment, cela suppose une connaissance éprouvée de sa tournée. Les clercs organisent en effet leur tournée sur la base des connaissances spatiales qu'ils ont pu acquérir sur leur environnement. On pourrait aussi parler de connaissances topographiques (le clerc expérimenté s'est en effet forgé une carte mentale très détaillée de son «territoire ») ou écologiques (dans la mesure où il a acquis des savoirs sur les

18. Cette caractéristique a été reprise par Béguin et Clot dans un article portant sur la place de l'action située dans le développement de l'activité (Béguin, \& Clot, 2004, op. cit.). 
rapports que les habitants entretiennent à leur quartier, à leur lieu de vie). Ces connaissances se rapportent à la configuration spatiale d'un quartier (agencement des rues, emplacement des bâtiments, présence ou pas de services publics), aux types de population qui habitent le quartier, aux rapports que les habitants entretiennent entre eux et avec leur espace, aux $u s$ et coutumes en vigueur dans l'environnement local, etc. Ces connaissances sont à la fois spatiales et sociales ${ }^{19}$, et relèvent d'un savoir clinique éprouvé (acquis au fil du temps). Elles sont situées et s'actualisent dans l'action et en situation. Elles permettent ainsi aux clercs titulaires de tournée d'organiser très rapidement leur tournée et d'optimiser leur trajet. En effet, contrairement aux « doubleurs », ils n'ont pas recours à un plan pour définir leur trajet et classent leur tournée très rapidement.

De plus, au moment de la préparation de la tournée, les clercs significateurs expérimentés mobilisent des ressources écrites. Il s'agit essentiellement d'informations retranscrites sur de petits carnets (des « carnets de tournée »). Sur ces carnets, sont par exemple consignés les numéros de téléphone de certains gardiens, les codes d'entrée de certains immeubles, des informations relatives à des bâtiments difficiles d'accès (ex: des informations sur les différentes entrées possibles pour pénétrer dans un bâtiment ), etc. Ainsi, quand ils préparent leur tournée, ils anticipent les difficultés qu'ils risquent de rencontrer dans la journée et vérifient qu'ils disposent bien des ressources qui leur permettront de surmonter ces difficultés. C'est aussi l'occasion pour eux d'actualiser certains renseignements consignés dans leurs carnets ${ }^{20}$ (notamment en fonction des dernières informations transmises par les études d'huissier).

Les clercs « énactent» et «asservissent» également leur environnement durant leur tournée en s'appuyant sur leurs compétences cognitives. Ces dernières renvoient à des modes de raisonnement, des procédés interprétatifs, des raccourcis cognitifs... qui sont mobilisés in situ (lors de leur travail d'enquête) et qui orientent l'action. Ces compétences cognitives renvoient notamment aux questions qu'ils se posent lorsqu'ils effectuent des enquêtes et aux modes de raisonnement sous-jacents à leur questionnement. Par exemple, si l'intéressé ne se trouve pas à son domicile et si personne ne peut ou ne veut recevoir l'acte, les clercs sont dans l'obligation de signifier l'acte en mairie, donc de se faire confirmer au préalable par une personne présente dans les lieux (un gardien, un parent, un voisin) le domicile de l'intéressé. Si ce dernier n'habite plus à son domicile, les clercs vont alors mener une véritable enquête, adopter un mode de raisonnement interrogatif et tenter de répondre à certaines questions types. La personne habite-t-elle toujours dans les lieux? Est-elle sur son lieu de travail? Est-elle partie en vacances? A-t-elle déménagé et si oui depuis quand est-elle partie? Quelle est sa nouvelle adresse? A-t-elle quitté les lieux sans laisser d'adresse? Rencontre-t-elle des problèmes financiers? Paye-t-elle régulièrement ses loyers ? Est-elle solvable? etc. Ces questions orientent leurs investigations et sont révélatrices du volet cognitif de leur activité.

Pour obtenir des réponses à leurs questions, les clercs significateurs disposent de trois types de ressources. Tout d'abord, ils sont guidés par les renseignements et les instructions qui ont été transmis par les études d'huissier ${ }^{21}$, mais encore faut-il savoir les décrypter et agir en conséquence. En cas de besoin, ils peuvent aussi contacter, via leur téléphone portable de service, l'huissier responsable du dossier, ou un de ses collaborateurs, pour obtenir des informations complémentaires. Ensuite, ils disposent sur le terrain de relais et peuvent s'adresser à des " personnes ressources », comme les gardiens d'immeuble. Ces derniers constituent des contacts d'autant plus précieux que les immeubles

19. Le social et le spatial sont en effet intimement liés, comme l'ont bien montré les sociologues de l'Ecole de Chicago (Burgess, \& Park, 1921; Park, 1952).

20. Certains disposent également d'un « cahier de déménagé » dans lequel ils consignent des informations relatives à des justiciables qui ont déménagé (ils indiquent notamment leur nouvelle adresse dans leur carnet). Ces renseignements, qui ont été recueillis dans le cadre de leur travail d'enquête, ou qui ont été transmis par une étude d'huissier, pourront leur être utiles ultérieurement. Un même justiciable peut en effet avoir affaire à plusieurs huissiers de justice. Les clercs ont donc tout intérêt à garder une trace de ce type d'information.

21. Les dossiers des justiciables sont gérés par les études. L'huissier contrôle et pilote (à distance) l'enquête. Celle-ci est donc une tâche partagée. Quand l'huissier décide d'externaliser la procédure de signification au Bureau, il ne délègue donc au clerc significateur qu'une partie du travail d'enquête (une autre partie est effectuée « en chambre » par l'huissier ou un de ses collaborateurs) 
sont de moins en moins accessibles; ils sont en effet souvent grillagés et protégés par des systèmes de sécurité électroniques (portes codées, badges, passes magnétiques, interphones ). Les gardiens ont donc la possibilité de donner aux clercs le code d'entrée de l'immeuble. Ils peuvent également leur ouvrir directement la porte et jouer un rôle d'informateur. Ils sont effectivement bien placés pour transmettre aux clercs des renseignements sur la situation d'un résident. L'extrait ci-dessous retrace un dialogue entre un clerc et une gardienne d'immeuble. Le clerc cherche à recueillir auprès de la gardienne des informations sur la situation de locataires pour procéder à une signification.

Le clerc: «Alors j'ai le 2182, 2182. Et ensuite j'ai celui là. Je suis passé l'autre fois, j'ai vu le remplaçant et lui il m'a dit qu'il gardait le courrier à la loge et il n'a pas pu me certifier le domicile ».

La gardienne: «Parce que heu. Ils avaient été expulsés et ils doivent récupérer l'appartement ». Le clerc: «Ah ils ont été expulsés. Ah ben voilà ! Et ils reviennent dans l'appartement. Et donc ils sont toujours locataires alors? Ils vont redevenir locataires. Ah j'ai le comment du pourquoi. Ils sont dans les lieux là actuellement? ».

La gardienne: «Y a personne. Ils le récupèrent cet après-midi ».

Le clerc: «Bon d'accord. Là je vais vous laisser un avis de passage. Là je vais voir s'il y a quelqu'un ».

La gardienne: «Lui je l'ai vu partir mais la dame là je la connais pas».

Le clerc: «Eux ils ont déjà eu des trucs ».

La gardienne: « Ouais ouais ouais ».

Le clerc: «Le $18^{\text {ème }}$ toujours? Ah ce sont des habitués eux hein?».

La gardienne: « Ouais».

Le clerc: «Allez au revoir et bonne journée ! ».

Enfin, si personne n'est présent dans les lieux, ou si personne n'est en mesure de les renseigner, les clercs chercheront dans leur environnement immédiat des informations susceptibles de les éclairer. L'intéressé a-t-il encore une boîte aux lettres à son nom à l'adresse indiquée ? Quel est l'état de sa boîte aux lettres? Son nom figure-t-il sur une liste affichée au mur ou à l'interphone ? Ce sont là autant d'indices, de signes, de traces... qui peuvent les guider dans leurs enquêtes. Les clercs agissent ainsi dans un environnement peuplé d'objets et de traces matérielles qui orientent leurs investigations. Ils constituent des «affordances » (Gibson, 1979), c'est-à-dire des prises pour l'action ${ }^{22}$. Mais encore faut-il être capable, en situation, de se poser les bonnes questions pour orienter son regard vers les objets pertinents et récolter des informations utiles... Ces informations, et les démarches entreprises par les clercs pour les besoins de leurs enquêtes, sont dans un second temps consignées dans des compte-rendus, qui sont ensuite transmis aux études d'huissier concernées. Ces compterendus, qui constituent des « écrits de travail situés » (Fraenkel, 2001), sont rédigés dans un style télégraphique et dans un langage qui n'est pas d'emblée compréhensible pour le profane. En effet, les mentions apposées sur les compte-rendus par les clercs - « domicile certain », «PSA»: parti sans laisser d'adresse, « mairie motivée », etc. - sont propres à un univers professionnel (celui des huissiers de justice et des clercs significateurs). Les clercs s'efforcent ainsi de transmettre aux études des informations factuelles, précises, objectives, et ce dans un style ramassé. L'extrait ci-dessous retrace un échange entre trois protagonistes (un clerc, une gardienne et son mari), et le commentaire fait par le clerc après-coup (au moment où il rédige une ébauche de compte rendu).

Le clerc ouvre la porte d'entrée. Un homme est sur le palier de la loge de la gardienne (il s'agit de son mari).

Le clerc: «Bonjour Monsieur! Je reviens comme la dernière fois pour un avis de quittance pour Monsieur X. J'ai sonné et il n'y a personne chez eux. Monsieur X c'est bien au deuxième étage c'est ça? ». [Le clerc cherche à se faire confirmer le domicile de Monsieur X]

L'homme: «Deuxième étage ».

Le clerc: «Elle est pas là votre épouse ? Non je crois qu'elle les prend pas. Posez-lui la question pour savoir si elle veut lui prendre. Demandez-lui si elle veut lui prendre ». [Le clerc veut savoir si

22. Pour Béguin et Clot (2004, op. cit.), l'idée maîtresse de Gibson (1979) est que nous percevons directement la valeur fonctionnelle des objets et leur signification pratique; la signification de l'objet est liée à l'expérience perceptuelle et à sa valeur praxique (à un objet correspond une signification pour l'action). 
la gardienne accepte de recevoir la copie de l'acte]

L'homme: «Je vais lui demander ».

Le clerc (s'adressant au chercheur) : «La dernière fois elle n'en a pas voulu. Alors je pense pas que Mais on va quand même lui demander dès fois que ça a changé ».

Le clerc: « Oui bonjour Madame. J'ai encore un pli pour Monsieur X ».

La gardienne: «Il n'est pas là. Alors je vais prendre l'avis ». [La gardienne refuse implicitement de prendre l'acte mais accepte de prendre l'avis de passage, avis qu'elle transmettra ensuite à la personne concernée]

Le clerc: «Vous prenez l'avis?».

La gardienne: « Ouais ouais!».

Le clerc remplit l'avis de passage.

La gardienne: «C'est encore pour une contravention?».

Le clerc: «Non non. Là c'est pour autre chose. C'est une procédure civile ».

La gardienne: «Il est parti ce matin ».

Le clerc: «Oui, ils sont partis tous les deux au travail ce matin. Bon vous voyez ça?».

La gardienne « Oui oui ».

Le clerc: «Allez bonne journée Madame».

Le clerc (commentant après-coup la scène) : « Bon là je retranscris. Je mets « absent » car il y a personne au domicile. Alors ensuite la loge Je fais un concentré: «La concierge m'a certifié le domicile ». Ensuite je mets : «Qui refuse la copie. Personne pour recevoir. Mairie motivée ». Voilà. Alors je ne mets pas tout tout de suite mais je mets les deux ou trois paramètres pour me souvenir de la situation ${ }^{23}$. Quand je connais un peu Il y a des endroits dont je me souviens et puis dès fois il y a des endroits que je ne connais pas. Ou alors je ne me souviens pas Bon cet immeuble je viens souvent parce qu'il y a des cabinets d'avocats ici. C'est un immeuble que je connais. Je connais la gardienne et tout ça. Mais il y a des immeubles où je vais une fois tous les ans ou tous les deux ans. Alors j'arrive et je ne peux pas me souvenir de l'immeuble. Là si je me souviens « 5 rue Marguerite » en arrivant chez moi je revois l'immeuble. Là j'arrive un petit peu à me resituer. Mais quand vous ne connaissez pas du tout vous n'arrivez plus à resituer l'endroit. C'est pourquoi il faut mettre un minimum d'indications ».

Cet extrait est révélateur du volet cognitif de l'activité des clercs significateurs. D'une part, il est illustratif de la démarche empruntée et du mode de raisonnement adopté pour procéder à la signification de l'acte authentique. Le clerc a ici appliqué la procédure de signification en suivant pas à pas les règles formelles en vigueur. Le mode de questionnement qu'il a adopté lui a permis de « dérouler » pas à pas la procédure de signification. Le clerc n'ayant pas pu procéder à une « signification à personne » (à la personne même de l'intéressé), dans la mesure où le justiciable n'était pas présent à son domicile et où personne ne lui a répondu, il a interrogé le mari de la gardienne pour savoir si la personne résidait bien à l'adresse indiquée (le clerc: « Je reviens comme la dernière fois pour un avis de quittance pour Monsieur X. J'ai sonné et il n'y a personne chez eux. Monsieur X c'est bien au deuxième étage c'est ça? »). Le mari de la gardienne ayant confirmé l'information détenue par le clerc, il a ensuite cherché à entrer en contact avec la gardienne pour procéder à la signification de l'acte authentique (le clerc: "Elle est pas là votre épouse? Non je crois qu'elle les prend pas. Posez-lui la question pour savoir si elle veut lui prendre »). Celle-ci ayant refusé de recevoir l'acte (elle s'est contentée de prendre l'avis de passage ${ }^{24}$ ), il ne lui restait plus qu'à procéder à une « signification en mairie ». La procédure de signification a ici été scrupuleusement respectée. D'autre part, le moment où il rédige son ébauche de compte-rendu est révélateur du chemin emprunté par le clerc pour synthétiser et traduire les informations clés extraites de la situation dans un langage professionnel (ex: «Personne pour recevoir», « Mairie motivée », etc.) et mémoriser la situation en vue de la rédaction ultérieure de son compte-rendu (le clerc: «(...) je mets les deux ou trois paramètres pour

23. En rentrant chez eux, les clercs complètent leurs compte-rendus (cela représente entre une heure et une heure et demie de travail par jour). Une fois les compte rendu complétés, ils sont ensuite transmis aux études d'huissier concernées (via le Bureau).

24. L'avis de passage qu'il laisse à la gardienne informe le justiciable de la situation. Sur présentation d'une pièce d'identité, ce dernier pourra venir retirer la copie de l'acte à la mairie de son domicile. 
me souvenir de la situation ». «Là si je me souviens « 5 rue Marguerite » en arrivant chez moi je revois l'immeuble »). Dans le cas présent, il peut d'autant plus facilement mémoriser la situation qu'il connaît l'immeuble concerné et les personnes rencontrées (la gardienne et son mari). La possession d'un certain nombre de connaissances spatiales facilite donc l'activité cognitive (notamment le processus de mémorisation ${ }^{25}$ ).

\section{Le rôle de la civilité dans l'activité des clercs significateurs}

Les rapports de civilité seront analysés dans une perspective goffmanienne. Goffman envisage l'interaction de face à face comme un domaine de plein droit, comme un type d'ordre social (Goffman, 1953), et la situation d'interaction comme un niveau d'analyse de la société. Selon lui, l'interaction relève d'un « ordre expressif » (Goffman, 1974, op. cit.). Ce dernier repose sur la préservation des «faces » : chaque acteur, dans ses échanges avec autrui, s'efforce de préserver sa face tout en maintenant celle des autres ${ }^{26}$ (Goffman, 1974, op. cit.). La civilité constitue ainsi un mécanisme régulateur de l'interaction sociale et contribue au maintien d'un certain ordre expressif.

La civilité occupe une place importante dans l'activité des clercs significateurs, notamment dans les contacts qu'ils ont avec les gardiens. Dans la mesure où ces derniers constituent des informateurs de première importance et qu'ils s'attendent à être traités avec respect ${ }^{27}$, les clercs s'efforcent d'entretenir de bonnes relations avec eux. Pour ce faire, ils n'hésitent pas à converser quelques instants avec eux et à investir dans les civilités d'usage. Ainsi, ils sont amenés à s'engager dans des conversations ordinaires, dans des discussions sans enjeu pour créer le contact ou préserver la relation. L'important est d'obtenir un consensus temporaire, un accord de surface (Goffman, 1973, op. cit.). Mais, dans le même temps, les clercs évitent d'entrer dans de longues discussions « consommatrices » de temps. Il $\mathrm{y}$ a donc un délicat équilibre à trouver entre engagement et distanciation.

Un clerc, évoquant les contacts qu'il entretient avec les gardiens de son secteur: «Il y a des fois où ils ont envie de papoter et on ne peut pas les envoyer bouler. Ou alors il faut faire ça de manière élégante. ( ). Trop connaître les gens dès fois c'est pas bon. Ca fait perdre du temps. C'est vrai qu'ils aiment bien profiter de ton passage pour bavarder un peu mais toi t'as pas le temps ».

Les clercs s'efforcent ainsi de créer un contexte relationnel favorable au déploiement de la procédure de signification. Leur objectif est in fine de recueillir des informations pertinentes auprès du gardien. Il s'agit en effet d'obtenir des renseignements supplémentaires et/ou de se faire confirmer une information qui est essentielle pour pouvoir procéder à la signification de l'acte authentique, ou tout du moins pour faire avancer une affaire. Les deux extraits ci-dessous mettent en évidence l'enchevêtrement des registres informatif et civil. Dans le premier cas, la civilité passe par le fait de prendre des nouvelles de son interlocuteur et d'accepter de converser quelques instants avec lui; dans le second, par un échange de plaisanteries. Les clercs significateurs s'appuient en fait sur des « ressources sûres » (Goffman, 1974, op. cit., 1953, op. cit.) pour créer un cadre propice à l'échange (pour « briser la glace » et instaurer une certaine convivialité) et recueillir les informations dont ils ont besoin ${ }^{28}$. Les codes de salutation, les rituels d'entrée en scène, les diverses manifestations de courtoisie, les phrases toutes faites, les «menus propos », les plaisanteries légères constituent autant de ressources sûres pour engager ou entretenir la conversation.

Extrait $n^{\circ} 1$ :

Un clerc (s'adressant à une gardienne): « Bonjour Julia! Tu vas bien? En forme? ». [Ici le clerc

25. On voit bien ici à quel point les compétences juridiques, cognitives et spatiales sont enchevêtrées.

26. «On peut définir le terme face comme étant la valeur sociale positive qu'une personne revendique effectivement à travers la ligne d'action que les autres supposent qu'elle a adoptée au cours d'un contact particulier » (Goffman, 1974, op. cit., p. 9).

27. Lorsqu'ils entrent en contact avec un gardien par le biais d'un interphone ou qu'ils frappent à la porte de sa loge, ils pénètrent sur un « territoire » qui est sous la responsabilité du gardien.

28. Dans sa thèse de doctorat, Goffman défini les ressources sûres comme des réserves de messages mobilisées par les individus quand ils sont dans une situation où ils doivent maintenir un échange (Goffman, 1953, op. cit.). 
investi dans le registre des civilités, ressource sûre]

Le clerc (montrant un pli à la gardienne): «Donc ça je sais, c'est chez les avocats, par contre ça ? ». [Demande d'information]

La gardienne (en regardant le nom écrit sur le pli) : «C'est en face des avocats ».

Le clerc: «C'est en face des avocats. Premier étage alors».

La gardienne : «Et toi comment vas-tu?». [Retour aux civilités d'usage]

Le clerc: « Ca va, ca va. J'attends les vacances. Et toi t'es pas parti là ?» (...).

Extrait $n^{\circ} 2$ :

La gardienne: « Bonjour!».

Le clerc (d'un ton enjoué): « Bonjour Madame la gardienne! Vous allez bien? J'ai sonné mais ça ne répond pas. Une famille Cyril, avec un C comme dans sauterelle ! Le numéro 324 ». [L'humour constitue ici une ressource sûre]

La gardienne: «Cyril Je le dépose dans la boîte?».

Le clerc: « Oui. Alors vous êtes contente de vous?».

La gardienne: « Non parce que mes locataires ils se plaignent sans arrêt et ça m'énerve !».

Le clerc: «Ah les méchants locataires!».

La gardienne: «Ah les méchants huissiers! ». [Eclat de rire des deux protagonistes. S'ensuit un échange de plaisanteries sur le rôle des huissiers].

Une dimension clé du travail de clerc significateur concerne les relations avec les justiciables. Les clercs doivent, à chaque fois, s'adapter à la situation et à leur interlocuteur pour trouver le bon positionnement. Il s'agit d'identifier (et ce « en un clin d'œil ») son interlocuteur, d'évaluer une situation et d'adopter un comportement approprié. En effet, comme l'a très bien analysé Goffman, il y a des choses que l'acteur peut ou ne peut pas se permettre au cours de sa « représentation » (Goffman, 1973, op. cit.). En la matière, tout dépend des personnes rencontrées, de leur statut et de leur comportement. Les relations avec les justiciables, bien qu'encadrées par un certain nombre de règles, restent pleines d'incertitudes. Cependant, les clercs s'appuient sur leurs compétences comportementales et relationnelles pour gérer « au mieux » les situations relationnelles (parfois délicates) dans lesquelles ils se trouvent placés. Les interactions entre les clercs et les justiciables peuvent être analysées à l'aune des travaux de Goffman sur la relation de service, dans la mesure où les registres technique, contractuel et civil (tels que définis par Goffman, 1968) s'entremêlent étroitement. Registre technique (ou informatif) tout d'abord: les clercs cherchent à se faire confirmer par les justiciables certains éléments (leur identité, leur adresse ) pour procéder à une « signification à personne ». Il s'agit de recueillir des informations objectives, factuelles. Registre contractuel ensuite: les justiciables doivent accepter de recevoir la copie de l'acte authentique qui leur est destinée. En effet, la « signification à personne » n'est possible que si ces derniers acceptent (explicitement ou implicitement) les termes du « contrat » (ils doivent confirmer leur nom, prénom, qualité, et accepter de recevoir la copie de l'acte en main propre). Registre civil enfin: les clercs investissent dans les civilités d'usage. Saluer son interlocuteur, faire preuve de politesse et de déférence, échanger une plaisanterie constituent autant de moyens de faciliter les interactions selon les deux autres registres. Ainsi, dans les relations avec les justiciables, les clercs significateurs font preuve de tact et de discrétion. C'est d'autant plus important que leur venue n'est que très rarement souhaitée. De ce fait, ils sont souvent vus comme des « porteurs de mauvaises nouvelles » pour reprendre les propos de certains d'entre eux. Le premier contact qui s'établit entre les clercs et les justiciables s'apparente à une épreuve pleine d'incertitudes. En effet, au moment où ils s'apprêtent à entrer en relation avec un justiciable, les clercs ne savent pas (ou rarement) à qui ils ont affaire. Ils ne connaissent généralement rien de la personnalité du justiciable et de son état d'esprit, pas plus qu'ils ne savent comment va réagir l'intéressé (il existe tout une gamme de comportements possibles qui vont de l'attitude coopérative à la colère en passant par l'indifférence ). En outre, pour réaliser une « signification à personne », les clercs sont amenés à rencontrer les justiciables à leur domicile. Dans une perspective goffmanienne, le domicile d'un justiciable constitue son « territoire personnel », le prolongement de son «Moi » ${ }^{29}$ (Goffman, 1974, op.

29. Dans la présentation qu'il fait de la sociologie goffmanienne, Isaac Joseph rappelle que chez Goffman le territoire 
cit.). Les clercs font donc irruption dans la vie privée des justiciables et, ce faisant, peuvent les mettre dans l'embarras. Dans certaines circonstances, les questions précises des clercs indisposent effectivement les justiciables. La situation peut alors devenir conflictuelle, comme dans l'extrait ci-dessous.

Le clerc est devant l'interphone de l'immeuble. Il sonne puis fait son entrée en scène en s'exprimant lentement et très distinctement: « Oui bonjour. Je vous prie de m'excuser. J'avais un courrier à remettre à Madame X de la part de la RIVP, pour Madame X. C'est bien ici ? » .

Un homme: « Oui ».

Le clerc: « Elle est là?».

L'homme: «Oui ».

Le clerc: «Alors je peux monter pour lui apporter le courrier? Au quatrième étage, c'est ça? ». La porte s'ouvre. Le clerc monte à l'étage, puis sonne à la porte. Un homme en peignoir ouvre la porte. Il semble mal réveillé.

Le clerc: «Bonjour Monsieur. Je vous prie de m'excuser. J'ai un pli à remettre à Madame X de la part de la RIVP».

L'homme: «Pardon?».

Le clerc (plus lentement): «J'ai un courrier pour Madame X».

L'homme: «De la part de qui ?».

Le clerc: « De la RIVP. C'est la société qui gère les logements ici. Elle n'est pas là? ».

L'homme: «Non, elle est partie au boulot».

Le clerc: «D'accord, je peux vous le laisser en son absence? Alors vous êtes Monsieur? ». [Il commence à « dérouler » la procédure de signification d'un ton neutre en demandant à l'homme ses nom, prénom et qualité]

L'homme: «Monsieur Z ».

Le clerc (d'un ton neutre) : «Et par rapport à cette dame, vous êtes un ami, un parent?».

L'homme (d'un ton sec): «Alors ça écoutez, ça ne vous regarde pas!».

Le clerc: « Mais je suis obligé de le marquer Monsieur! ». [Le clerc cherche à se justifier]

L'homme: «Alors écoutez, soit vous gardez votre courrier et ».

Le clerc (en lui coupant la parole et en lui tendant l'avis de passage): « Pas de problème Monsieur.

Tenez vous donnerez ça à Madame ». [Il essaye d'éviter la polémique]

L'homme: «Je donne rien! Il y a le concierge! ». Il claque la porte.

Le clerc laisse l'avis de passage sous la porte. La porte s'ouvre à nouveau (brusquement).

L'homme: «De toute façon moi j'ai rien vu!».

Le clerc: «Moi non plus Monsieur. Pas de problème ».

Le clerc (qui commente la situation tout en établissant son compte rendu): « Je me suis présenté à l'étage. Il m'a confirmé le domicile. Est-ce que j'ai pu donner le pli à la dame ? Non, elle n'était pas là. Je lui ai posé la question pour savoir s'il en voulait. Qu'est-ce qu'il m'a répondu ? Non. On résume. Alors ce que je mets sur le compte rendu. On a des compte rendu types Et donc « Domicile certain. Absent. Une personne dans les lieux refuse de recevoir la copie. Mairie motivée ». Je ne le fais pas pour me faire plaisir parce qu'il n'y avait personne mais c'est parce qu'on me l'a refusé. Là, on me l'a refusé catégoriquement. Il m'a dit qu'il ne le voulait pas ( ). Je sais très bien que c'est son ami dans la mesure où il arrive en robe de chambre mais il faut qu'il me le dise ».

Ici, le clerc ne peut finalement pas procéder à une « signification à personne ». En effet, la personne refuse catégoriquement de coopérer; elle montre même une certaine agressivité. Dès lors, il est dans l'impasse; il ne peut pas « dérouler » la procédure de signification et doit donc procéder à une signification « en mairie ». Dans son compte rendu, il fait part des démarches qu'il a entreprises, des informations recueillies in situ et justifie sa position.

Pour prévenir l'embarras des justiciables et le cortège d'émotions qui l'accompagne, les clercs significateurs doivent faire un travail de « figuration », au sens où l'entend Goffman: «Par figuration (face-work) j'entends désigner tout ce qu'entreprend une personne pour que ses actions ne fassent

est un « concept emprunté à l'éthologie qui désigne l'espace fixe, situationnel ou personnel sur lequel un ayant droit exerce un contrôle et dont il défend les limites » (Joseph, 1998, op. cit., p. 124). 
perdre la face à personne (y compris elle-même). La figuration sert à parer aux « incidents », c'est-àdire aux événements dont les implications symboliques sont effectivement un danger pour la face » (Goffman, 1974, op. cit., p. 15). Les clercs sont ainsi amenés à s'engager dans un travail de figuration pour « dédramatiser » les situations. Cela passe notamment par l'usage de «procédés préventifs » (Goffman, 1973, op. cit.). L'usage de tels procédés leur permet de prévenir les tensions inhérentes aux échanges avec les justiciables, de ne pas entamer une discussion qui porterait sur le fond de l'affaire (donc de ne pas polémiquer), et d'écourter la conversation ${ }^{30}$. L'enquête a mis en évidence plusieurs procédés préventifs.

Tout d'abord, les clercs significateurs s'efforcent d'être informatifs, précis et concis. Pour bien se faire comprendre de leurs interlocuteurs, ils s'expriment en général lentement et très distinctement (c'est d'autant plus vrai lorsqu'ils nouent un premier contact avec une personne par le biais d'un interphone comme dans l'extrait présenté précédemment) et ne donnent aux justiciables qu'un minimum d'informations. Ainsi, ils ne commentent pas le contenu de l'acte (pour ne pas indisposer les personnes rencontrées et éviter toute polémique) et peuvent ne dévoiler la provenance de l'acte qu'à partir du moment où les justiciables le leur demandent expressément (comme dans l'extrait ci-dessus). Pour éviter toute discussion, certains vont même jusqu'à affirmer qu'ils ne connaissent pas la provenance de l'acte et restent laconiques (voir l'extrait ci-dessous).

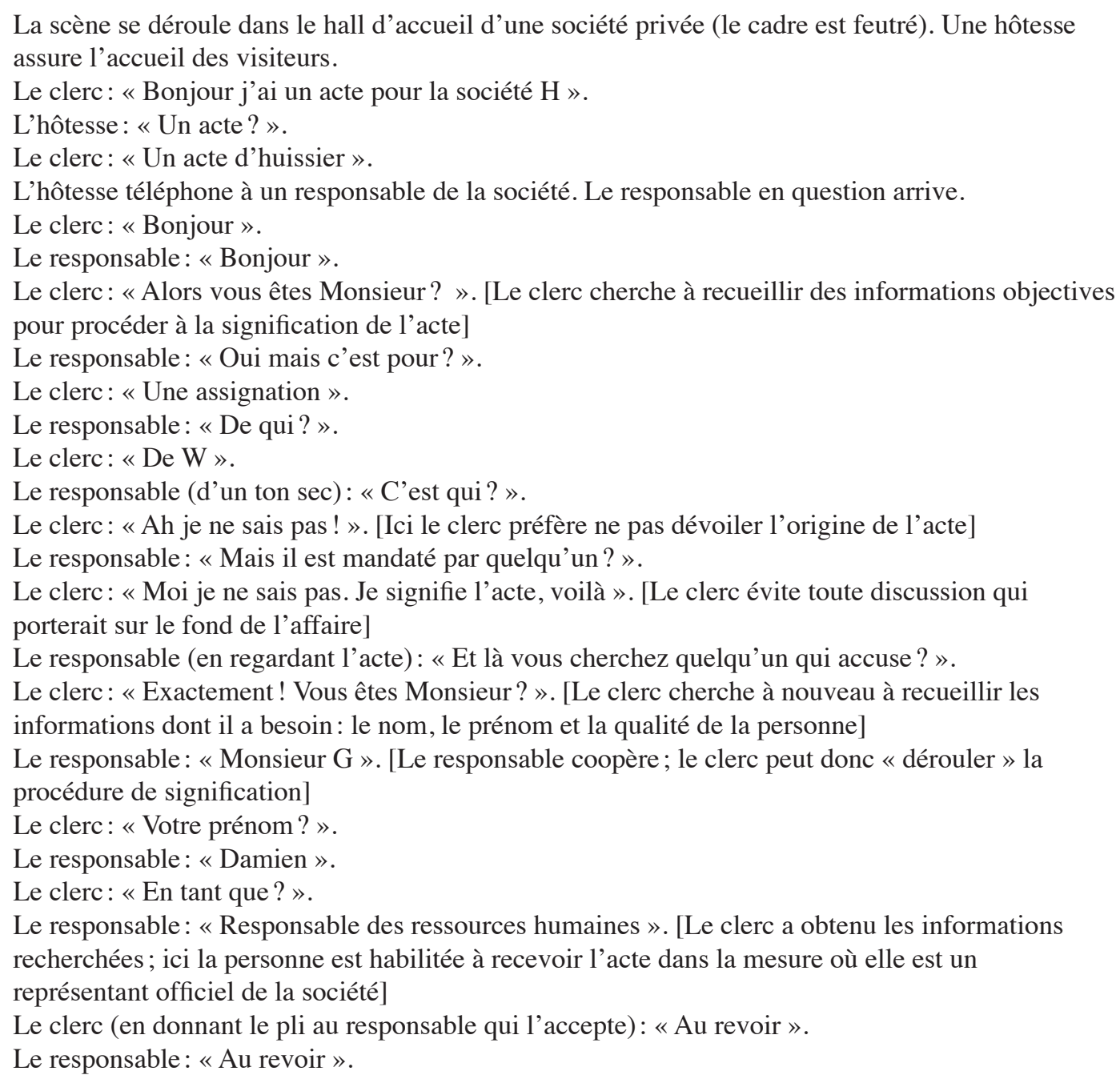

Dans cet exemple (cas d'une signification à personne morale), le clerc cherche à signifier au plus vite.

30. Il faut également rappeler qu'une partie de la rémunération des clercs significateurs du Bureau (la partie variable) dépend du nombre d'actes qu'ils délivrent. Ils sont donc incités à signifier dans les plus brefs délais. 
Il ne donne en effet à son interlocuteur que les informations qui lui sont nécessaires pour que ce dernier comprenne et accepte la situation. La tactique adoptée par le clerc est de ne pas en rajouter dans les solennités requises et de faire en sorte que l'action menée s'insère dans le cours des événements. Les clercs significateurs s'efforcent d'ailleurs d'être perçus par les justiciables comme de simples intermédiaires (c'est le cas dans l'exemple ci-dessus), comme des « porteurs de plis », des « facteurs », ou encore des « coursiers » pour reprendre les propos de certains.

Un clerc: «Moi de n'ai qu'un contact avec les gens. Je ne les connais pas. Je travaille pour eux, mais je ne les connais pas. Moi je leur dis que je suis comme le facteur. Si le facteur vous apporte vos impôts ou un avis de décès Moi j'apporte le courrier. Mais il y en a qui ne comprennent pas ».

Ensuite, les clercs significateurs se gardent de tout jugement de valeur, ne s'immiscent jamais dans la vie privée des gens, et adoptent une mise en scène corporelle appropriée. Ainsi, quand ils procèdent à une signification au domicile des justiciables, ils s'efforcent de respecter leur territoire personnel, restent systématiquement sur le pas de la porte, à une distance raisonnable de leur interlocuteur (ni trop près pour ne pas violer leur espace personnel, ni trop loin dans la mesure où il s'agit d'entendre et d'être entendu), et évitent les contacts oculaires appuyés (susceptibles de mettre la personne dans l'embarras). Il s'agit là d'autant de marques de civilité.

Enfin, ils font preuve de tact et de discrétion pour « dédramatiser » la situation quand un public assiste à la scène. Ils évitent en effet que leur interlocuteur ne perde la face devant leur public (famille, amis, clients..). C'est notamment le cas lorsque les clercs signifient un acte authentique auprès d'un commerçant. Ils doivent en effet être discrets pour ne pas mettre le commerçant concerné en porteà-faux vis-à-vis de sa clientèle. La présence de clients dans le magasin impose donc une certaine réserve et l'adoption d'un comportement approprié, comme dans l'extrait ci-dessous.

La scène se déroule dans une boulangerie. Le boulanger est derrière son comptoir. Ce dernier sert les quelques clients qui sont là.

Le clerc: « Bonjour».

Le chercheur: «Bonjour».

Le clerc: « On est ensemble. J'ai un commandement qui vient de Silico ».

Le boulanger semble étonné: « Silico? Je connais pas».

Il regarde l'acte.

Le clerc: « Tu connais?».

Le boulanger: «Ah oui d'accord! ».

Le boulanger (interpellant le clerc): «Les deux plis que tu m'as amenés l'autre semaine, c'est réglé ».

Le clerc: «C'est réglé. Bonne journée. Au revoir».

Le clerc (commentant la scène): « Mais là tu vois comme c'est un commerçant je le fais toujours discrètement. Parce que pour la clientèle il faut pas que j'arrive comme un sagoin. On se met dans un petit coin et on parle. Tu vois? Il y a des choses qui ne se font pas. Parce que nous aussi par rapport à cette clientèle, il y a des choses qui ne se font pas. Il faut adopter un comportement approprié ».

\section{Perspectives et retombées pratiques de cette étude}

Au-delà du fait que cette étude apporte un éclairage sur un métier mal connu - celui de clerc significateur - en quoi peut-elle contribuer au développement de l'activité de signification des actes authentiques et améliorer le mode de fonctionnement des bureaux de signification $?^{31}$.

Une des particularités de l'activité de signification des actes authentiques est son caractère solitaire.

31. Bien que le gérant du Bureau n'attendait pas de retombées opérationnelles immédiates de cette enquête, il a cependant transmis à ses confrères huissiers de justice - qui sont également ses « clients » (tout du moins ceux qui sollicitent les services du Bureau) - un rapport de recherche intermédiaire rédigé dans la foulée de l'enquête. En diffusant très largement ce rapport, l'objectif du gérant était de valoriser le travail des clercs significateurs et de mettre en avant l'utilité du Bureau pour la profession d'huissier de justice. 
En effet, les clercs significateurs passent la plus grande partie de leur temps seuls, à distance du Bureau, de leur hiérarchie et de leurs collègues. Cet isolement a été pensé et organisé par le Bureau, de même que les modalités de contrôle et d'évaluation de leur travail. Chaque clerc a ainsi son territoire, est autonome dans le cadre de sa tournée, est évalué sur le nombre d'actes signifiés (dans la journée ou dans le mois), et peut être audité à tout moment par sa hiérarchie (qui peut diligenter une enquête interne en cas de problème $)^{32}$.

Bien que cette solitude soit la conséquence de choix organisationnels décidés en amont, mais aussi une forme de réponse au désir d'indépendance et d'autonomie des clercs significateurs ${ }^{33}$, elle n'est pas sans poser problème sur le plan du développement et de la transmission des compétences. En effet, dans le meilleur des cas, les clercs ont suivi un enseignement théorique relatif à la procédure de signification des actes authentiques ${ }^{34}$, pris connaissance du manuel du clerc significateur, et reçu une formation pratique pour faciliter leur prise de poste (dispensée « sur le tas » par un « chef de secteur »). Mais dans la mesure où le développement et la transmission des compétences n'ont pas véritablement été pensés par le gérant et l'encadrement du Bureau ${ }^{35}$ (contrairement aux procédures d'évaluation et de contrôle de l'activité), les nouveaux venus dans la profession sont condamnés à apprendre l'essentiel de leur métier seuls et dans l'action, et le développement de leurs compétences est contingent aux situations (plus ou moins formatrices) qu'ils rencontrent sur le terrain et aux liens qu'ils nouent avec leurs collègues de travail ${ }^{36}$.

Il s'agirait donc de faire du développement et de la transmission des compétences une réflexion partagée au sein de la structure (par le gérant, l'encadrement, les organisations syndicales et l'ensemble des clercs). Une telle réflexion collective aurait le mérite d'interroger les modalités de management et de gestion des ressources humaines existantes pour les orienter davantage vers le développement et la transmission des compétences ${ }^{37}$.

Un référentiel de compétences et de ressources professionnelles spécifique au métier de clerc significateur, construit dans une logique participative, pourrait constituer un objectif opérationnel à moyen terme. Le processus d'identification et de formalisation des compétences et des ressources professionnelles propres à la pratique de signification des actes authentiques permettrait aux professionnels concernés de travailler sur le cœur du métier de clerc significateur pour en dégager les « fondamentaux $»^{38}$, de repérer et de capitaliser les «bonnes pratiques », et de disposer d'un support pour la formation des nouveaux entrants dans la profession.

L'enquête a mis en évidence quatre types de connaissances et/ou de compétences: (1) les connaissances juridiques (qui renvoient à la procédure de signification des actes authentiques); (2) les

32. Il s'agit donc d'une évaluation et d'un contrôle a posteriori.

33. En effet, les clercs apprécient tout particulièrement l'autonomie dont ils bénéficient dans leur travail.

34. Cet enseignement est dispensé par l'Ecole Nationale de Procédure et comporte 6 modules et 9 jours en centre de formation. Au programme des notions de droit, des procédures et de la communication: délivrer l'acte; gérer la tournée; aviser le destinataire des conséquences de l'acte; régulariser l'acte; contrôler et mettre en forme l'acte; enquêter sur la solvabilité.

35. Le gérant et l'encadrement ont privilégié un apprentissage «sur le tas » (sous l'égide d'un clerc plus expérimenté), mais le processus et les conditions de cet apprentissage n'ont pas fait l'objet d'une réflexion spécifique.

36. On peut faire l'hypothèse que l'intensité de ces liens dépend beaucoup de la personnalité du novice, du bon vouloir de ses collègues de travail (de leur volonté de transmettre ou pas les « ficelles » du métier...), du type de relation qui se noue entre le novice et ses collègues plus expérimentés, de l'ambiance de travail au sein du groupe et de l'attitude du « chef de secteur »...

37. Dans la mesure où les modalités de management et de gestion des ressources humaines sont avant tout basées sur une logique de contrôle et sur une culture de la méfiance (les enquêtes internes réalisées par l'encadrement visent avant tout à débusquer les «tricheurs »), elles ne peuvent pas réellement constituer des leviers d'action en matière de développement et de transmission des compétences.

38. C'est d'autant plus important, qu'au moment de l'enquête, les fiches de poste qui circulaient dans l'organisation étaient très anciennes (certaines d'entre elles avaient été tapées à la machine à écrire !) et qu'elles étaient quasiment vides en terme de contenu. En outre, il n'existait dans l'organisation aucun descriptif d'activité (référentiel...). Le seul document en prise avec la pratique de signification des actes authentiques et décrivant le métier de clerc significateur était le manuel du clerc significateur. 
connaissances et compétences spatiales (mobilisées à la fois lors de la préparation de la tournée et lors des tournées); (3) les compétences cognitives (mobilisées par les clercs dans le cadre de leur travail d'enquête et qui renvoient à des questionnements spécifiques); (4) les compétences relationnelles (notamment en matière de civilité). Évidemment, ces connaissances et compétences, qui sont dans la pratique étroitement enchevêtrées, mériteraient d'être discutées par les acteurs concernés. Cette typologie constitue ainsi une base de dialogue susceptible d'encourager la confrontation des points de vue et d'alimenter une réflexion collective.

En outre, un certain nombre de ressources peuvent être répertoriées. Hormis les ressources officielles qui sont données aux clercs significateurs par le Bureau, par des organismes de formation, ou par des études d'huissier - comme par exemple le manuel du clerc significateur, les connaissances juridiques transmises par les formateurs de l'Ecole Nationale de Procédure, les outils confiés aux clercs (téléphone portable, scooter ou mobylette, fiches de compte rendu, etc.), les renseignements transmis par les études et qui portent sur la situation de certains justiciables... - il existe de nombreuses ressources officieuses. Ces dernières constituent des « ressources opératoires » au sens où l'entend Céline Chatigny $(2001)^{39}$. Certaines sont partagées et d'autres individuelles. Par ailleurs, elles sont hétérogènes. En effet, en situation, les clercs s'appuient sur un ensemble de ressources techniques, matérielles, écrites et humaines. Sans prétendre à un inventaire exhaustif de ces ressources, rappelons celles qui ont déjà été évoquées plus haut : - la pratique de la tournée en « arc de cercle » (technique connue par les plus anciens dans le métier); - les objets pertinents (sources d'informations) que les clercs identifient dans leur environnement immédiat lors de leurs tournées (boîtes aux lettres, interphones, listes des résidents affichées dans les halls d'immeubles...); - les « carnets de tournée » (sur lesquels sont inscrits notamment les numéros de téléphone de certains gardiens et les codes d'entrée de certains immeubles); - les «cahiers de déménagé » (sur lesquels sont consignées des informations relatives à des justiciables qui ont déménagé); - les « personnes ressources », notamment les gardiens d'immeuble (les clercs expérimentés se sont en effet constitués un véritable réseau d'informateurs). Ces ressources, qui sont mobilisées par les clercs les plus expérimentés, semblent circuler difficilement dans l'organisation ${ }^{40}$. En effet, elles sont propres à des individus ou à des collectifs restreints (par exemple des clercs expérimentés qui ont intégré le Bureau à la même époque).

Dès lors, on peut s'interroger sur les conditions à mettre en place pour favoriser la transmission de ces ressources (des experts vers les novices) et soutenir le processus d'acquisition de connaissances et de compétences professionnelles. En effet, ces ressources issues de la pratique n'ont de valeur et d'efficacité qu'en contexte. Elles ne constituent des ressources opératoires qu'à partir du moment où elles sont utiles à la pratique et que les novices sont engagés dans une dynamique d'acquisition et de développement de compétences professionnelles. Par exemple, la tournée « en arc de cercle » est une technique, une ficelle du métier, qui ne prend sens que dans la pratique. Cette pratique repose elle-même sur la mobilisation de certaines connaissances et compétences (notamment spatiales) ${ }^{41}$.

Plusieurs pistes sont envisageables pour améliorer le partage de ressources et de compétences professionnelles:

- bâtir en concertation avec les acteurs intéressés par la démarche un référentiel de compétences et de ressources professionnelles spécifique au métier de clerc significateur, puis diffuser systématiquement ce référentiel auprès des nouveaux entrants dans la profession;

— intégrer ce référentiel dans la formation pratique destinée à faciliter la prise de poste (il pourrait

39. Pour Chatigny, ces ressources opératoires sont « des aides que les individus se construisent individuellement et collectivement, à partir d'éléments de l'environnement de travail et de vie privée représentant pour eux une source potentielle de ressources » (Chatigny, 2001, op. cit., p. 8).

40. Le fait que les clercs agissent seuls, à distance du Bureau, et qu'il n'existe pas réellement d'espaces de discussion dans l'organisation expliquent pour partie cette situation. Le moment de la préparation de la tournée, le matin dans la grande salle du Bureau et les lieux d'échange informels, comme les cafés par exemple, semblent être les seuls espaces de discussion...

41. En effet, l'appropriation de la technique de la tournée « en arc de cercle » n'est possible que si le clerc a une connaissance éprouvée de sa tournée. 
s'agir d'un outil utile aux « chefs de secteur » qui viendrait en complément du manuel du clerc significateur);

- instaurer un dispositif de formation plus formel et plus collectif lors de la prise de poste en faisant jouer aux clercs les plus expérimentés un rôle de formateur pour initier les novices aux « fondamentaux » du métier et diffuser les ressources issues de la pratique (celles-ci pourraient être présentées en lien avec des cas concrets ${ }^{42}$ );

- mettre en place un dispositif fondé sur les retours d'expérience pour lutter contre la solitude dans le métier et permettre aux clercs d'engager des retours réflexifs sur leur activité, et pour capitaliser les «bonnes pratiques » et susciter des apprentissages collectif et organisationnel ${ }^{43}$.

- Il s'agit là d'exemples de dispositifs qui sont de nature à consolider le métier - ils peuvent en effet aider à l'émergence d'un « genre du métier » ${ }^{44}$ (Clot, \& Faïta, 2000, op. cit.) - et à renforcer la professionnalité des clercs significateurs.

\section{Conclusion}

La procédure in abstracto, c'est-à-dire celle qui est déposée sur un support écrit et qui apparaît sous la forme d'un corpus de règles formelles et prescriptives, " impose » une trame générale qu'il convient de suivre dans toutes les situations et le respect d'un certain nombre d'étapes et de séquences d'action. Mais la procédure ne dit rien, ou presque, sur les conditions de son application à des cas concrets; elle est d'une certaine manière « muette » et se caractérise par son incomplétude. Il revient ainsi aux agents qui sont chargés de suivre la procédure de la « compléter » (Castel, \& Merle, 2002; Reynaud, 2001, 2005). Pour ce faire, ils s'appuient non seulement sur un ensemble de règles formelles définies par la procédure, mais aussi sur des éléments puisés dans la situation et sur leurs compétences. La mise en œuvre de la procédure de signification dans des situations toujours singulières (la procédure « en acte ») ne tient donc que grâce au travail de "petites mains »- les clercs significateurs - et aux compétences qu'ils mobilisent in situ. Les règles liées à la procédure de signification ne déterminent donc pas entièrement leur conduite. Pour pouvoir « dérouler » la procédure, les clercs doivent aussi s'adapter au contexte, aux circonstances et saisir les opportunités qui s'offrent à eux dans leur environnement. En définitive, suivre une règle est une pratique, comme l'a bien montré Wittgenstein (Wittgenstein, 1958). En effet, dès lors que l'on adopte une approche pragmatique des règles et que l'on envisage celles-ci, non pas de manière abstraite, mais de manière concrète, à travers leurs usages, on se rend compte que l'application des règles dans des situations particulières incorpore un ensemble d'éléments hétérogènes issus à la fois de l'environnement et de l'expérience professionnelle.

\section{RÉFÉRENCES}

Bancaud, A. (1989). Une « constance mobile »: la haute magistrature. Actes de la recherche en sciences sociales, 76-77, 30-48.

Barnes, B. (1995). The Elements of Social Theory. London: UCL Press.

Béguin, P., \& Clot, Y. (2004). L'action située dans le développement de l'activité. Activités, 1(2), $27-49$. http://www.activites.org/v1n2/beguin.fr.pdf

Bernard, S. (2005). Le temps de l'activité de la caissière: entre logique productive et logique de service. Sociologie du Travail, 47(2), 170-187.

42. Cela supposerait évidemment d'élaborer une banque de cas pertinents...

43. L'apprentissage organisationnel supposerait la construction d'une «mémoire organisationnelle ». Cette mémoire organisationnelle serait de nature à améliorer le mode de fonctionnement collectif de l'organisation en faisant évoluer les pratiques des acteurs concernés.

44. Dans la mesure où les clercs significateurs sont isolés et qu'ils n'ont pas réellement l'occasion de confronter leurs expériences, il n’existe pas réellement de « genre du métier ». 
Bittner, E. (1967). The Police on Skid-Row: A Study of Peace Keeping. American Sociological Review, 32(5), 699-715.

Bourdieu, P. (1986). La force du droit. Eléments pour une sociologie du champ juridique. Actes de la recherche en sciences sociales, 64, 3-19.

Burgess, E.-W., \& Park, R.-E. (1921). Introduction to the Science of Sociology. Chicago: University of Chicago Press.

Burns, S. (1997). Practicing Law: A Study of Pedagogic Interchange in a Law School Classroom ». In M. Travers M., \& J. Manzo (Eds.), Law in Action. Ethnomethodological and Conversation Analytic Approaches to Law (pp 265-287). Aldershot: Ashgate.

Caroly, S., \& Weill-Fassina, A. (2007). En quoi différentes approches de l'activité collective des relations de services interrogent la pluralité des modèles de l'activité en ergonomie ? Activités, 4 (1), 85-98. http:// www.activites.org/v4n1/v4n1.pdf

Castel, P., \& Merle, I. (2002). Quand les normes de pratiques deviennent une ressource pour les médecins. Sociologie du travail, 44(3), 337-355.

Cerf, M., \& Falzon, P. (2005). Situations de service : travailler dans l'interaction. Paris: PUF (Coll. Travail Humain).

Chatigny, C. (2001). Les ressources de l'environnement: au cœur de la construction des savoirs professionnels en situation de travail et de la protection de la santé. PISTES, 3(2). http://www.pistes. uqam.ca/v3n2/pdf/v3n2a7.pdf

Cicourel, A. (1995). The Social Organization of Juvenile Justice. New Brunswick and London: Transaction Publishers.

Clot, Y., \& Faïta, D. (2000). Genres et styles en analyse du travail. Concepts et méthodes. Travailler, 4, 7-42.

Collard, D. (2003). Analyser les compétences des médiateurs dans les gares de banlieue. Travail et emploi, 94, 37-43.

Deharo, G. (2005). Ce qu'exécuter veut dire Une approche théorique de la notion d'exécution. Droit et procédures, 4, 208.

Dejours, C. (1995). Le facteur humain. Paris: PUF.

Dejours, C. (1998). Souffrance en France. Paris: Éditions du Seuil.

Demazière, D., \& Mercier, D. (2003). La tournée des facteurs : normes gestionnaires, régulation collective et stratégies d'activité. Sociologie du Travail, 45(2), 237-258.

Denis, J. (2007). La prescription ordinaire. Circulation et énonciation des règles au travail. Sociologie du Travail, 49(4), 496-513.

Dingwall, R., \& Greatbatch, D. (1994). Divorce mediation - the Virtues of Formality. In J. Eekelaar, \& M. Maclean (Eds.), Oxford Readings in Socio-Legal Studies: Family Law (pp 391-399). Oxford: Oxford University Press.

Djibo, S. (2008). Contribution d'une analyse du discours à l'étude des stratégies d'actions et de régulation des agents en situation d'écoute téléphonique : l'exemple du dispositif d'urgence sociale 115. Activités, 5(1), 3-20. http://www.activites.org/v5n1/v5n1.pdf

Dupret, B. (2001). Le droit en action et en contexte. Ethnométhodologie et analyse de conversation dans la recherche juridique. Droit et Société, 48, 343-348.

Falzon, P., \& Lapeyrière, S. (1998). L'usager et l'opérateur: ergonomie et relations de service. Le Travail humain, 61(1), 69-90.

Favret-Saada, J. (1978). Les mots, la mort, les sorts. Paris: Gallimard.

Fraenkel, B. (2001). La résistible ascension de l'écrit au travail. In A. Borzeix A., \& B. Fraenkel (Eds.), Langage et Travail. Communication, cognition, action (pp 113-142). Paris: CNRS Éditions. 
Fraenkel, B. (resp. scientifique), Pontille, D., Collard, D., \& Deharo, G. (2005). Pratiques juridiques et écrit électronique : le cas des huissiers de justice. Rapport de recherche, Paris: Mission de recherche « Droit \& Justice », GIP du Ministère de la Justice.

Fraenkel, B. (resp. scientifique), Pontille, D., Collard, D., Deharo, G., \& Blanchette, J.F. (2004). Pratiques juridiques et écrit électronique : la signature dans la société de l'information. Rapport de recherche, Paris: programme CNRS « Société de l'information».

Gadrey, J. (1994). Les relations de service et l'analyse du travail des agents. Sociologie du Travail, 3, 381390.

Garcia, A. (1991). Dispute Resolution without Disputing: How the Interactional Organisation of Mediation Hearings Minimizes Argument. American Sociological Review, 56(6), 818-835.

Garfinkel, H. (1948). A Research Note on Inter- and Intra- Racial Homicides. Journal of Social Forces, 27(4), 369-381.

Garfinkel, H. (1967a.). Studies in Ethnomethodology. Englewood Cliffs, NJ: Prentice-Hall.

Garfinkel, H. (1967b). Practical sociological reasoning: some features in the work of the Los Angeles Suicide Prevention Center. In E.-S. Schneidman (Ed.), Essays in Self-Destruction (pp 171-187). New York: International Science Press.

Gibson, J.J. (1979). The ecological approach to visual perception. Hillsdale: Lawrence Erlbaum associates.

Goffman, E. (1953). Communication Conduct in an Island Community. Thèse de doctorat, Chicago: Département de sociologie, Université de Chicago.

Goffman, E. (1968). Asiles, Études sur la condition sociale des malades mentaux. Paris: Les Éditions de Minuit.

Goffman, E. (1973). La mise en scène de la vie quotidienne, t.1 : La présentation de soi. Paris: Les Éditions de Minuit.

Goffman, E. (1974). Les rites d'interaction. Paris: Les Éditions de Minuit.

Guinchard, S., \& Moussa, T. (2005). Droit et pratiques des voies d'exécution. Paris: Dalloz.

Guinot, T. (2004). L’huissier de justice. Paris: EJT.

Halperin, J.L. (1996). Avocats et notaires en Europe. Les professions judiciaires et juridiques dans l'histoire contemporaine. Paris: LGDJ (Coll. Droit et Société).

Isnard, J. (2004). Le destin de la signification: entre méthodes d'aujourd'hui et techniques de demain. Droit et procédure, 1 .

Joseph, I. (1995). Métiers du public : les compétences de l'agent et l'espace de l'usager. Paris: CNRS Éditions.

Joseph, I. (1998). Erving Goffman et la microsociologie. Paris: PUF (Coll. Philosophies).

Karpik, L. (1995). Les avocats. Entre l'Etat, le public et le marché. XIIIe - XXe siècle. Paris: Gallimard.

Kirsh, D. (1995). The intelligent use of space. Artificial Intelligence, 73(1-2), 31-68.

Komter, M.L. (1998). Dilemmas in the Courtroom. Mahwah: Lawrence Erlbaum Associates.

Latour, B. (2002). La fabrique du droit. Une ethnographie du Conseil d'État. Paris: Éditions la Découverte.

Leblanc, S., Ria, L., Dieumegard, G. Serres G., \& Durand M. (2008). Activités, 5(1), 58-78. http://www. activites.org/v5n1/v5n1.pdf

Leduc, S. (2003). L'accueil dans les bureaux de poste. Approche dynamique des compétences et de l'organisation du travail dans les relations de service. Thèse de doctorat, Amiens: Université de Picardie Jules Verne.

Leplat, J. (2004). - Éléments pour l'étude des documents prescripteurs. Activités, 1(2), 195-216. http://www. activites.org/v1n2/Leplat.pdf 
Licoppe, C. (2002). Le traitement des courriers électroniques dans les centres d'appels. Sociologie du Travail, $44(3), 381-400$.

Losego, P. (2004). Le travail invisible à l'université : Le cas des antennes universitaires. Sociologie du Travail, 46(2), 187-204.

Mathieu-Fritz, A. (2003). Les représentations sociales de la profession d'huissier de justice. Droit et Société, 54, 491-517.

Mathieu-Fritz, A. (2005). Les Huissiers de justice. Paris: PUF (Coll. Sciences sociales et Sociétés).

Mathieu-Fritz, A. (2006). Les mutations de la profession d'huissier de justice depuis les années 70. Sociologie du Travail, 48(1), 55-71.

Maynard, D. (1984). Inside Plea-Bargaining : The Language of Negotiation. New York/London: Plenum Press.

Merton, R. (1965). Éléments de théorie et de méthode sociologique. Paris: Plon.

Milburn, P. (2002). La compétence relationnelle: maîtrise de l'interaction et légitimité professionnelle: avocats et médiateurs. Revue française de sociologie, 43(1), 47-72.

Park, R.E. (1952). Human Communities. The city and Human Ecology. Glencoe: Free Press.

Parsons, T. (1968). Professions. International Encyclopedia of the Social Sciences, 12, 536-547.

Quemin, A. (1997). Les commissaires-priseurs. La mutation d'une profession. Paris: Éditions Anthropos.

Relieu, M., Salembier, P., \& Theureau, J. (2004). Introduction au numéro spécial « Activité et Action/ Cognition Située ». Activités, 1(2), 3-10. http://www.activites.org/v1n2/intro.pdf

Reynaud, B. (2001). « Suivre des règles » dans les organisations. Revue d'Economie Industrielle, 97, 53-68.

Reynaud, B. (2005). Une approche Wittgensteinienne des règles économiques. Revue de Métaphysique et de Morale, 47(3), 349-374.

Salembier, P., \& Zouinar, M. (2004). Intelligibilité mutuelle et contexte partagé. Inspirations conceptuelles et réductions technologiques. Activités, 1(2), 64-85. http://www.activites.org/v1n2/salembier.pdf

Suchman, L. (1987). Plans and Situated Actions, The Problem of Human Machine. New York: Cambridge University Press.

Sudnow, D. (1965). Normal Crimes : Sociological Features of the Penal Code in a Public Defender Office. Social Problems, 12, 255-276.

Suleiman, E. (1987). Les notaires. Les pouvoirs d'une corporation. Paris: Le Seuil.

Theureau, J. (1992). Le cours d'action: analyse sémiologique. Berne: Peter-Lang.

Theureau, J., \& Filippi, G. (1994). Cours d'action et conception d'une situation d'aide à la coordination: le cas de la régulation du trafic du RER. Sociologie du Travail, 36(4), 547-562.

Theureau, J., Filippi, G., Saliou, G., \& Vermersch, P. (2002). Cultural issues of nuclear power plant collective control in accidental situations and their impact upon design issues. In Proceedings of the Eleventh European Conference on Cognitive Ergonomics - ECCE 11. Catania, Italy, 8-11 septembre.

Thuderoz, C. (1991). Notaires et huissiers de justice: du patrimoine à l'entreprise. Revue française de sociologie, 32(2), 209-239.

Tilinski, Y. (2002). La responsabilité professionnelle de l'huissier de justice. Thèse de doctorat, Marseille: Université d'Aix-Marseille.

Travers, M. (1997). The reality of Law. Work and Talk in a firm of Criminal Lawyers. Aldershot: Ashgate.

Travers, M., \& Manzo, J.F. (1997). Law in Action. Ethnomethodological and Conversation Analytic Approaches to Law. Aldershot: Ashgate. 
Valléry, G. (2002). L'ergonomie dans la dynamique d'étude des situations de travail en relation de service. Dimensions interactives des activités, perspectives organisationnelles, développement des compétences professionnelles. Habilitation à diriger des recherches, Amiens: Université de Picardie Jules Verne.

Valléry, G. (2004). Relations de service et approche ergonomique: saisir le caractère dynamique et situé de l'activité au travers de l'analyse des interactions «agent-client ». Activités, 1(2), 121-146. http://www. activites.org/v1n2/vallery.pdf

Valléry, G., \& Bonnefoy, M-A. (1997). La relation de service dans les organismes publics à caractère social : entre le dire et le faire de l'agent. Performances Humaines et Techniques, 89, 15-25.

Varela, F. J. (1989). Autonomie et connaissance. Essai sur le vivant. Paris: Le Seuil.

Weick, K-E. (1977). Enacment Processes in Organizations. In B.-M. Staw, \& G.-R. Salancik (Eds.), New Directions in organizational behaviour (pp. 267-300). Chicago: St. Clair Press.

Weick, K-E. (1988). Enacted Sensemaking in Crisis Situation. Journal of Management Studies, 25(4), 112127.

Wittgenstein, L. (1958). Philosophical Investigations. Oxford: Basil Blackwell.

\section{RÉsumé}

Cet article montre que le suivi d'une procédure ne résulte pas de la procédure elle-même. L'application d'une procédure est une activité qui passe par une série d'accomplissements pratiques réalisés en situation (qui ne peuvent pas être définis ex ante). Le propos est illustré à partir du cas de la mise en ouvre d'une procédure juridique appelée « signification des actes authentiques » réalisée par les clercs d'huissier de justice. Si cet acte juridique impose une trame générale qu'il convient de suivre dans toutes les situations, l'observation montre que les clercs doivent néanmoins, dans la pratique, compléter la règle, gérer les contingences locales et saisir les opportunités présentes dans leur environnement. Ceci amène à distinguer la procédure in abstracto - comme corpus de règles formelles déposé sur un support écrit - et la procédure « en acte » - comme ensemble d'actions engagées dans une situation particulière et qui prennent appui sur des ressources hétérogènes: des normes écrites, des données puisées dans le contexte local, une expérience et des compétences professionnelles.

\section{MoTS CLÉS}

Signification des actes authentiques, procédure, pratique, compétences, situation.

\section{RÉFÉRENCEMENT}

Collard, D. (2010). De la procédure in abstracto à la procédure «en acte» : le cas de la signification des actes authentiques par les clercs d'huissier de justice. Activités, 7(1), pp. 111-135, http://www.activites.org/ v7n1/v7n1.pdf

Article soumis le 22 Juin 2009, accepté pour publication le 12 février 2010 